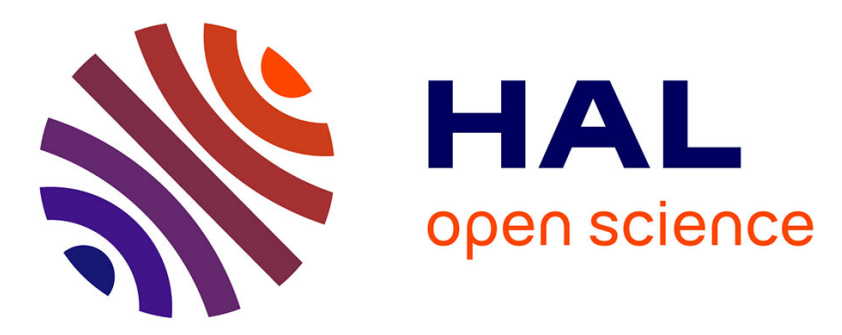

\title{
Le trésor de deniers mérovingiens de Rodez (Aveyron). Circulation et diffusion des monnayages d'argent dans le Sud de la France au milieu du VIIIe siècle

\author{
Vincent Geneviève, Guillaume Sarah
}

\section{- To cite this version:}

Vincent Geneviève, Guillaume Sarah. Le trésor de deniers mérovingiens de Rodez (Aveyron). Circulation et diffusion des monnayages d'argent dans le Sud de la France au milieu du VIIIe siècle. Revue Numismatique, 2010, 166, pp.477-507. 10.3406/numi.2010.2947 . hal-00579633

\section{HAL Id: hal-00579633 \\ https://hal.science/hal-00579633}

Submitted on 4 Feb 2020

HAL is a multi-disciplinary open access archive for the deposit and dissemination of scientific research documents, whether they are published or not. The documents may come from teaching and research institutions in France or abroad, or from public or private research centers.
L'archive ouverte pluridisciplinaire HAL, est destinée au dépôt et à la diffusion de documents scientifiques de niveau recherche, publiés ou non, émanant des établissements d'enseignement et de recherche français ou étrangers, des laboratoires publics ou privés. 


\section{Le trésor de deniers mérovingiens de Rodez (Aveyron).}

\section{Circulation et diffusion des monnayages d'argent dans le Sud de}

\section{la France au milieu du VIIle siècle}

\section{Vincent Geneviève, Guillaume Sarah}

\section{Résumé}

En 2002, un petit trésor de neuf deniers mérovingiens a été découvert dans une sépulture à Rodez (Aveyron, France). L'étude numismatique et les analyses élémentaires de composition des alliages permettent d'envisager son enfouissement dans les années 750-760, soit une date charnière entre la fin du pouvoir mérovingien et l'avènement du pouvoir carolingien.

\section{Abstract}

In 2002, a small hoard comprising nine Merovingian deniers has been discovered in a burial at Rodez (Aveyron, France). The combination of numismatic study and elementary analysis seems to indicate a concealment date circa 750-760 which is the transitional period between the Merovingians and the Carolingians.

\section{Citer ce document / Cite this document :}

Geneviève Vincent, Sarah Guillaume. Le trésor de deniers mérovingiens de Rodez (Aveyron). Circulation et diffusion des monnayages d'argent dans le Sud de la France au milieu du VIIle siècle. In: Revue numismatique, 6e série - Tome 166, année 2010 pp. 477-507;

doi : https://doi.org/10.3406/numi.2010.2947

https://www.persee.fr/doc/numi_0484-8942_2010_num_6_166_2947

Fichier pdf généré le 18/01/2019 


\section{Le trésor de deniers mérovingiens de Rodez (Aveyron). Circulation et diffusion des monnayages d'argent dans le Sud de la France au milieu du viII ${ }^{\mathrm{e}}$ siècle $^{1}$}

Résumé - En 2002, un petit trésor de neuf deniers mérovingiens a été découvert dans une sépulture à Rodez (Aveyron, France). L'étude numismatique et les analyses élémentaires de composition des alliages permettent d'envisager son enfouissement dans les années 750-760, soit une date charnière entre la fin du pouvoir mérovingien et l'avènement du pouvoir carolingien.

Summary - In 2002, a small hoard comprising nine Merovingian deniers has been discovered in a burial at Rodez (Aveyron, France). The combination of numismatic study and elementary analysis seems to indicate a concealment date circa 750-760 which is the transitional period between the Merovingians and the Carolingians.

L'opération archéologique «Jacobins II» à Rodez (Aveyron) s'est déroulée en deux phases, de mars à juillet 2001 puis de juillet à août 2002, préalablement à la construction d'une nouvelle école de musique et d'un parking souterrain dans le cœur historique de la ville ${ }^{2}$. Depuis la première campagne de fouilles conduite sur la place Raynaldy entre 1990 et 1992, il était connu que ce nouveau projet culturel s'installait à l'emplacement du forum antique et de l'ensemble conventuel des Jacobins (figure 1).

* Institut National de Recherches Archéologiques Préventives, ZI Les Pinsons, 13 rue du Négoce, F-31 650 Saint-Orens-de-Gameville ; chercheur associé, ITEM-GRA (EA 3002), Université de Pau et des Pays de l'Adour. Courriel : vincent.genevieve@inrap.fr.

** Institut de Recherches sur les Archéomatériaux - Centre Ernest Babelon, CNRS, Université d'Orléans, 3D rue de la Férollerie, F-45071 Orléans Cedex 2. Courriel : guillaume.sarah@cnrsorleans.fr.

1. Nous tenons ici à exprimer nos sincères remerciements à $\mathrm{M}$. Bompaire $(\mathrm{CNRS})$ et $\mathrm{Br}$. Foucray (SRA d'Ile-de-France) pour leurs précieux conseils, M. Amandry et D. Hollard (BnF) pour nous avoir facilité l'accès aux plateaux des monnaies mérovingiennes du Cabinet des Médailles de Paris, Fr. Planet (Musée des Beaux-Arts de Lyon) et S. Frey-Kupper (Avenches) pour les clichés photographiques et la documentation qu'ils nous ont transmis, L. Grimbert (INRAP) qui a bien voulu nous confier cette étude et enfin J. Lafaurie $(\dagger)$ qui a accepté de nous recevoir en octobre 2003, de discuter avec nous de ce trésor et surtout de nous encourager à en assurer la publication.

2. L. Grimbert et al., Rodez. Les Jacobins II. Document Final de Synthèse, Service Régional de l'Archéologie de Midi-Pyrénées, Toulouse, 2003, 3 vol. 


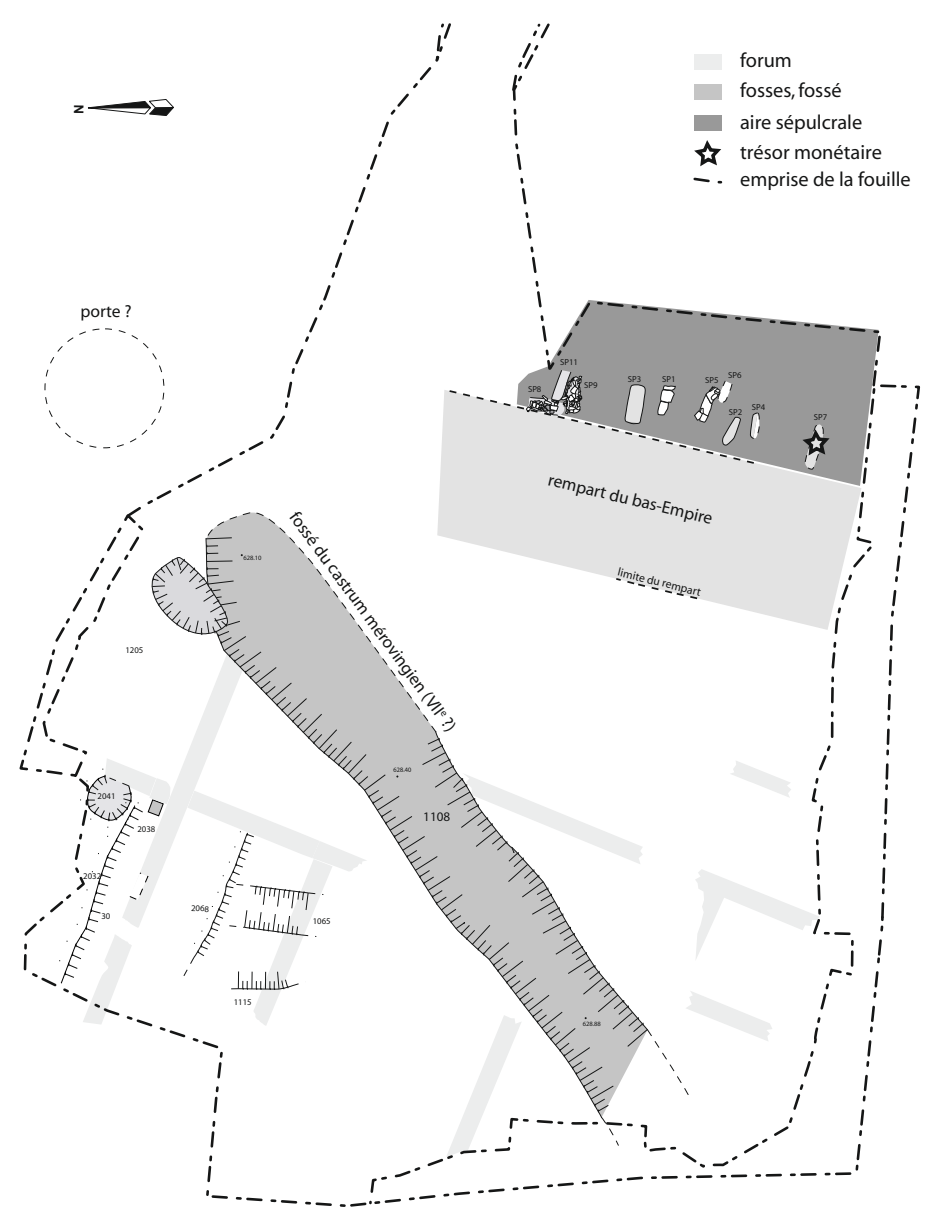

Figure 1 - Les niveaux du Haut Moyen Âge et le cimetière mérovingien du site des Jacobins à Rodez (infographie : L. Grimbert, INRAP).

Cette perspective laissait donc augurer de nouvelles découvertes importantes pour la capitale ruthène ${ }^{3}$. Ainsi, parmi les apports majeurs de cette nouvelle fouille, on relève notamment l'extension maximale, à son angle nord-est, du forum romain du milieu du $\mathrm{I}^{\mathrm{er}}$ siècle ap. J.-C., la localisation précise du rempart du Bas Empire ainsi que la découverte d'un petit cimetière mérovingien inédit adossé contre cette enceinte. L'emprise totale de l'aire sépulcrale reste inconnue en raison des limites imposées par la fouille et seuls quelques individus, dix exactement, ont pu être soigneusement dégagés.

3. J. CATALO, Rodez : du forum antique au couvent des Jacobins, Aquitania, VIII, 1990, p. 161-186. 
Le trésor monétaire a été découvert dans la sépulture d'une femme âgée entre 25 et 50 ans. Composé de neuf deniers déposés sous le genou gauche de l'individu, ce trésor constitue le seul mobilier recueilli dans la tombe. Aucun contenant n'a été remarqué, mais le regroupement des monnaies permet de supposer qu'elles étaient enfermées dans une bourse en matériau périssable, vraisemblablement en cuir ou en tissu, voire même se trouver directement cousues dans un vêtement. Aussi maigre que soit ce pécule, la quantité de pièces qu'il réunit le qualifie de trésor et le fait entrer dans un cercle très restreint de trouvailles de monnaies d'argent mérovingiennes découvertes sur le sol français depuis deux siècles.

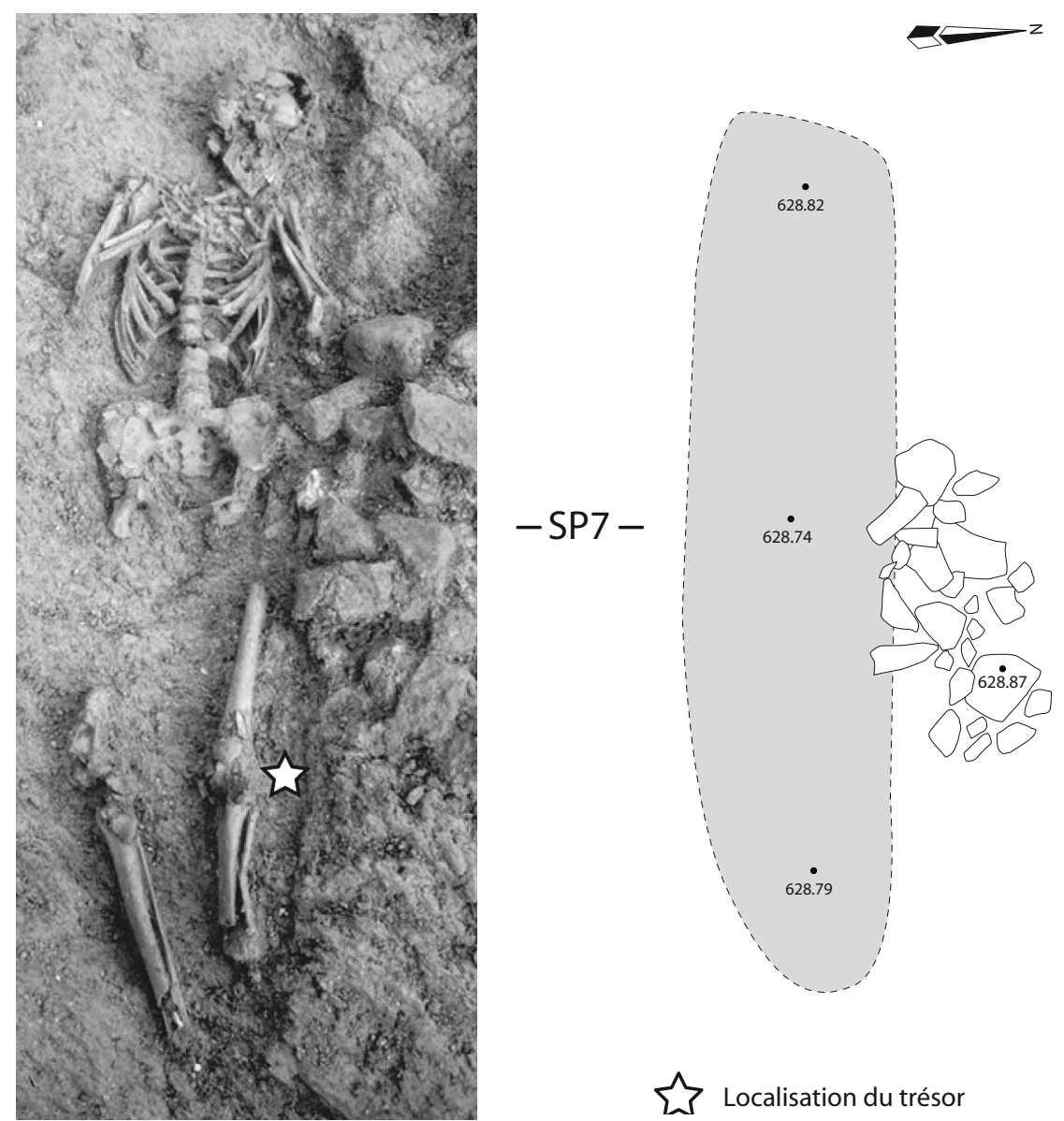

Figure 2 - La sépulture 7 et le trésor monétaire (infographie : L. Cordier et L. Grimbert, INRAP). 


\section{Une découverte rarissime}

En 1996, J. Lafaurie proposait, dans son chapitre consacré aux deniers mérovingiens de la collection du musée des Beaux-Arts de Lyon $^{4}$, une estimation d'environ 3500 exemplaires connus. Cette évaluation ne méconnaît pas les limites qui sont les siennes : celles du recensement exhaustif des collections publiques nationales, des découvertes fortuites qui nous sont parvenues ${ }^{5}$, ainsi qu'une simple approximation du potentiel des collections privées pour lesquelles toutes données précises échappent, ou presque, aux numismates ${ }^{6}$.Afin de mieux apprécier la portée de ces chiffres, signalons que le trésor de Nice-Cimiez (AlpesMaritimes) $)^{7}$ découvert en 1851 totalisait à lui seul au moins 2294 deniers, soit près des deux tiers des exemplaires qui nous sont actuellement connus. À cette trouvaille exceptionnelle, s'ajoutent les dépôts plus modestes de Saint-Pierre-lesÉtieux (Cher, 104 ex.), Plassac (Gironde, 177 ex.), Nohanent (Puy-de-Dôme, env. 27-35 ex. $)^{8}$, Saint-Jean-d'Heurs (Puy-de-Dôme, 30 ex. $)^{9}$, Savonnières (Indre-et-Loire, 45 ex.) ${ }^{10}$, Bais (Ille-et-Vilaine, 380 ex.) ${ }^{11}$ et Barbuise (Aube, 9 ex. $)^{12}$ qui représentent environ 780 deniers supplémentaires.

4. J. LAFAurie, Monnaies mérovingiennes du musée des Beaux-Arts de Lyon, Bulletin des musées et monuments lyonnais, supplément 1-2, Lyon, 1996, p. 40.

5. De très nombreuses trouvailles de sites sont recensées dans J. Lafaurie, J. Pilet-Lemière, Monnaies du Haut Moyen Âge découvertes en France ( $V^{e}-v_{I I I}^{e}$ siècle), Cahiers Ernest Babelon, 8 , Paris, 2003. Ce catalogue, on ne peut plus précieux, ne constitue malheureusement qu'un état des lieux de ces trouvailles si l'on considère le nombre d'exemplaires qui apparaissent quotidiennement depuis quelques années sur de multiples sites internet.

6. À ce titre, le musée Saint-Raymond-musée des Antiques de Toulouse a pu récemment acquérir auprès d'un particulier un lot d'une quinzaine de deniers mérovingiens dont une fraction, au moins, appartenait sans aucun doute au trésor de Nice-Cimiez. Achetées à l'extrême fin du XIX siècle ou au tout début $\mathrm{du} \mathrm{xx}^{\mathrm{e}}$, puis transmises au sein de la famille du collectionneur de génération en génération, ces monnaies semblent, à l'exception de deux exemplaires, totalement inconnues des inventaires actuels. L'étude de ce lot est actuellement en cours.

7. A. Сhaboullnet, Catalogue raisonné de la collection de deniers mérovingiens des vire et VIII ${ }^{e}$ siècles de la trouvaille de Cimiez donnée au Cabinet des Médailles par Arnold Morel-Fatio, Paris, 1890.

8. J. LAFAURIE, Monnaies d'argent mérovingiennes des VII et VIII ${ }^{\mathrm{e}}$ siècles : les trésors de SaintPierre-les-Étieux (Cher), Plassac (Gironde) et Nohanent (Puy-de-Dôme), RN, 1969, p. 98-219, pl. XV-XXI.

9. Ce trésor n'est connu qu'au travers d'une correspondance ancienne, voir J. LAFAURIE, Ibid., p. 113.

10. J. LAFAuRIE, Trésor de deniers mérovingiens trouvé à Savonnières (Indre-et-Loire), $R N$, 1963, p. 67-81, pl. VIII.

11. J. LAFAURIE, Nouvelle édition du catalogue des deniers mérovingiens de la trouvaille de Bais (Ille-et-Vilaine) de Prou et Bougenot, avec nouvelle préface, additions, réattributions et illustrations, Paris, 1981.

12. J. LAFAURIE, Deniers d'argent mérovingiens trouvés à Barbuise (Aube), BSFN, 43, 1988, p. 390-395 pour l'étude la plus récente. 
Parmi ces rares trouvailles disséminées sur tout le territoire, le trésor de Rodez constitue un document numismatique de première importance et ce pour plusieurs raisons. Sa composition, qui recèle plusieurs exemplaires inédits, augmente les corpus existants de nouvelles occurrences. Notamment, un denier parisien jusqu'alors inconnu contribue à compléter la chronologie des évêques de la ville et permet de dater assez précisément l'enfouissement du dépôt. Enfin, la localisation de ce trésor dans une région qui n'est pas une de celles qui produisent beaucoup de monnaies d'argent à cette période n'est pas sans susciter quelques interrogations.

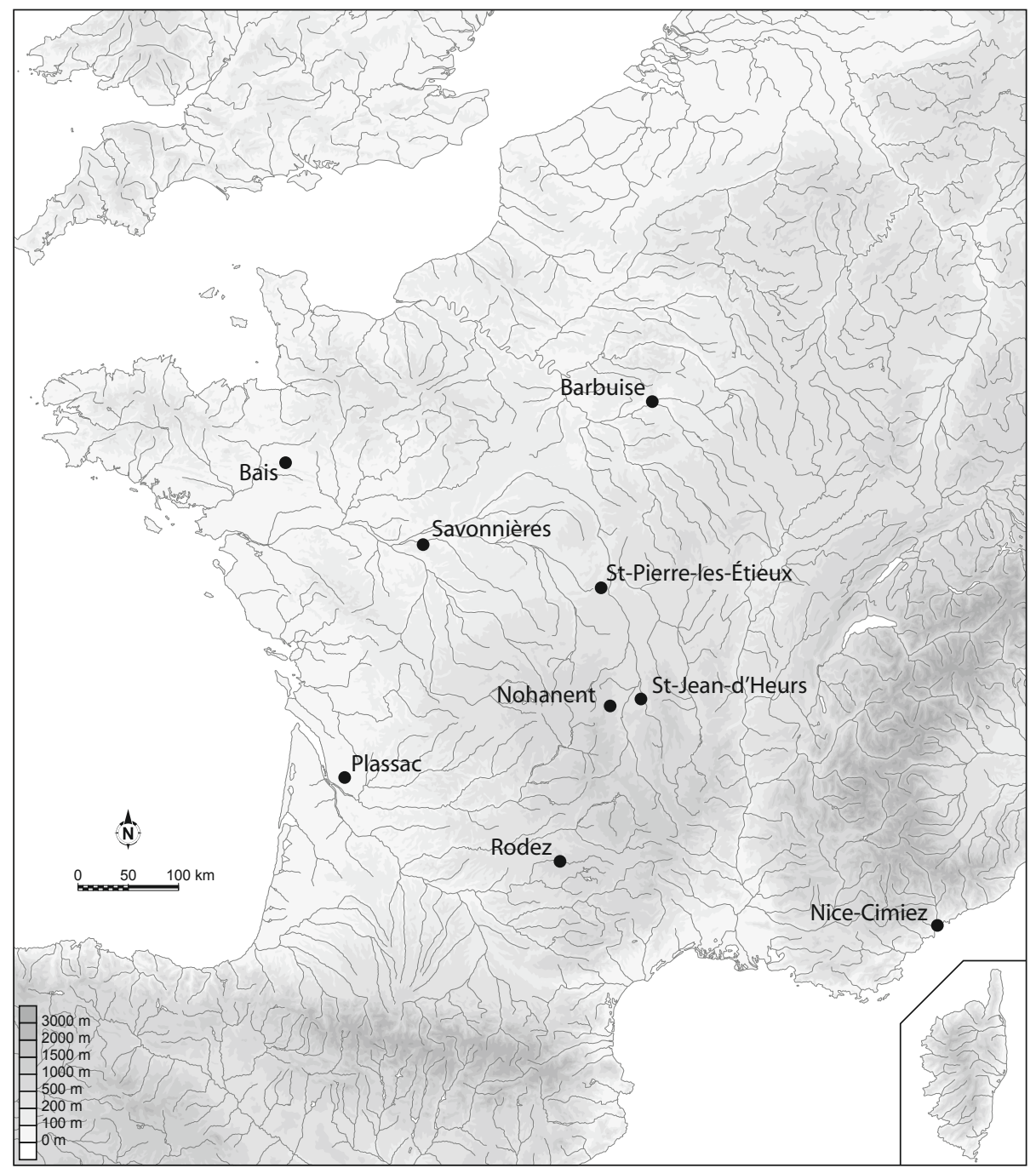

Figure 3 - Les trésors de deniers mérovingiens découverts en France. 


\section{Principes généraux}

L'introduction du denier mérovingien dans la circulation monétaire intervient autour des années 675-680. Le nom de la monnaie nous est connu grâce à la mention denarius sur quelques légendes : on relève notamment les mentions LVGDVNO DINARIOS sur un denier frappé à Lyon ${ }^{13}$ et DINARI AVRILIAI sur un autre émis à Orléans ${ }^{14}$. La date de sa création peut être précisée par un exemplaire frappé dans l'atelier de Tours et trouvé à Onzain (Loir-et-Cher) au nom de Childéric II, qui règne de 663 à 675, et que Jean Lafaurie propose de dater des années $673-675^{15}$. Ces deniers sont, dans la majorité des cas, connus au travers des trésors, les trouvailles isolées s'avérant peu courantes ${ }^{16}$. Leurs légendes se caractérisent, comme pour les derniers tremisses d'or, par un nom de lieu associé à celui d'un personnage identifié comme étant un monétaire ou une personnalité en charge de la frappe. La masse théorique de ces deniers se situe autour de 1,35 g, soit une taille qui correspond au 1/240 de la livre. Leurs émissions interviennent après une période longue de près de trois siècles presque exclusivement dominée en Gaule par la frappe de monnaies d'or dont la production semble s'arrêter brusquement dans le dernier quart du vII ${ }^{\mathrm{e}}$ siècle. En effet, aucun trésor mixte associant séries monétaires en or et en argent, n'est signalé à ce jour. On relèvera simplement la présence d'un tremissis en or pâle frappé à Banassac (Lozère) parmi la centaine d'exemplaires en argent du trésor de Saint-Pierre-les-Étieux ${ }^{17}$. Quant à la production des deniers mérovingiens, elle cesse sous le règne de Pépin le Bref (751-768) après l'avènement des premiers Carolingiens.

\section{Catalogue commenté du trésor}

Le catalogue des deniers du trésor de Rodez suit le classement proposé par A. de Belfort ${ }^{18}$, complété par ceux de M. Prou ${ }^{19}$ et de G. Depeyrot ${ }^{20}$ ainsi que par l'étude des collections et trésors précédemment cités. Les datations de tous ces exemplaires sont dans l'ensemble incertaines mais quelques indices

13. BELFORT 2352, Prou 989.

14. Belfort 542, Prou - .

15. J. LAFAURIE, Denier de Childéric II trouvé à Onzain (Loir-et-Cher), BSFN, 3, 1965, p. 512514 ; Un nouveau denier de Childéric II attribuable à Tours, $B S F N, 7,1988$, p. 421-426; À propos des deniers de Tours à la titulature de Childéric II, BSFN, 9, 1988, p. 462-464.

16. Environ 200 exemplaires si l'on se réfère à la recension la plus récente de J. LAFAURIE, J. Pilet-Lemière, op. cit., n. 5.

17. J. Lafaurie, op. cit., n. 8, p. 115, cat. 56 (Belfort 759, Prou 2107).

18. A. DE Belfort, Description générale des monnaies mérovingiennes, Paris, 5 vol., 1892-1895.

19. M. Prou, Catalogue des monnaies françaises de la Bibliothèque nationale. Les monnaies mérovingiennes, Paris, 1892.

20. G. Depeyrot, Le numéraire mérovingien. L'âge du denier, Moneta, 22, Wetteren, 2001. 
chronologiques permettront d'avancer une datation possible pour l'enfouissement de ce dépôt ${ }^{21}$.

\section{Briossus, Brioux-sur-Boutonne (Deux-Sèvres)?}

Anépigraphe ; tête à g. fruste résultant de l'utilisation d'un coin bouché.

Anépigraphe ; croix grecque fendue aux quatre extrémités et cantonnée de quatre globules ayant pour centre un annelet qui contient un globule.

Poids 0,90 g ; diam. 11-10 mm ; Belfort 978-979 ; Prou 2300-2303 ; Depeyrot 1, p. 103.

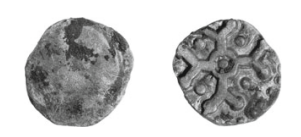

Ce type monétaire est recensé dans le trésor de Nice-Cimiez ${ }^{22}$. Malgré son droit fruste, cet exemplaire est aisément identifiable par la croix très caractéristique du revers que l'on retrouve antérieurement sur certains tremisses portant la légende de cet atelier ${ }^{23}$. Néanmoins, on notera que de tels deniers ne se retrouvent pas sur les sites poitevins et que de récentes analyses isotopiques réalisées sur un exemplaire du trésor de Nice-Cimiez n'ont pas révélé une quantité d'argent de Melle aussi importante qu'on aurait pu l'attendre compte tenu de la proximité immédiate de cet atelier ${ }^{24}$. Le droit est presque lisse mais si la lecture est juste, on semble encore distinguer les vestiges d'une tête orientée à gauche avec la main devant le visage. Ce denier pose clairement le problème plusieurs fois évoqué pour les frappes mérovingiennes, qu'elles soient d'or ou d'argent, de l'utilisation des coins monétaires jusqu'à la limite de leur usure. Parmi les hypothèses formulées pour tenter de répondre à cette question, la pénurie de main-d'œuvre, particulièrement de graveurs, ou celle d'alliages spéciaux pour la confection des coins, pourrait justifier cette utilisation intensive des matrices jusqu'à leur rupture ${ }^{25}$. Deux autres exemplaires du trésor (cat. 7 et 9) présentent la même caractéristique.

21. Deniers du trésor de Rodez: photos J.-Fr. Peiré (DRAC Midi-Pyrénées) ; denier d'Ins (Suisse) : photos B. Redha (Service archéologique du canton de Berne) ; denier conservé au musée de Lyon: photos A. Basset (Musée des Beaux-Arts de Lyon); deniers conservés au cabinet des Médailles de la BnF : photos V. Geneviève.

22. A. Morel-Fatio, dans A. Chaboulllet, op. cit., n. 7, p. 43, cat. 188, pl. IX, no 174.

23. Voir notamment les exemplaires BELFORT 975 et 976.

24. A. Clairand, Fl. Téreygeol, L'atelier monétaire mérovingien de Melle (Deux-Sèvres) : premières conclusions, dans A. Clairand, D. Hollard, Numismatique et archéologie en PoitouCharentes, Actes du colloque de Niort, 7-8 décembre 2007, Paris, 2009, p. 31-47 et plus particulièrement, p. 33 et 45 .

25. J. LAFAURIE, op. cit., n. 8, p. 127. 


\section{Brivas, Brioude (Haute-Loire)}

...V...X-... ; dans le champ, BR[ sous un tilde surmonté d'une croix.

Traces de légende ?; pentagone en forme de mitre rempli de globules. Éléments de titulature autour?

Poids 0,80 g ; diam. 10-8 mm ; Belfort 1018 ; Prou 1804-1805; Depeyrot 2 et s., p. 121-123.

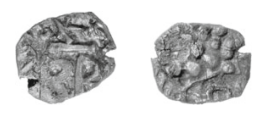

Ce type monétaire est recensé dans le trésor de Nice-Cimiez ${ }^{26}$. L'attribution de ce denier à Brioude ne pose a priori pas de problème si tant est que l'interprétation de ce monogramme soit juste et ne renvoie pas à un autre centre émetteur comme la question se pose pour l'exemplaire précédent.

\section{Arvernus ?, Clermont (Puy-de-Dôme) ?}

Monogramme rétrograde formé des lettres $\mathrm{AR}$ (avec une barre verticale à d. du A), ou NAR ou VAR, surmonté d'un tilde dans un champ de points.

Deux oiseaux affrontés et inversés dans un champ de points ; la tête d'un troisième, orientée à d., apparaît sous les pattes de l'un d'eux.

Poids 0,99 g ; diam. 12-11 mm ; Belfort - ; Prou - ; Depeyrot -.

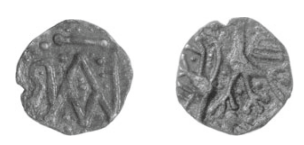

Des représentations d'oiseaux apparaissent sur de nombreux tremisses mérovingiens mais aucune ne s'apparente, tant dans la composition du thème que dans le style, à celle figurant sur ce denier ${ }^{27}$. L'attribution de cet exemplaire inédit à Clermont s'appuie sur plusieurs éléments, essentiellement d'ordre stylistique. Ainsi, la composition du monogramme, même en gravure rétrograde, est très fidèle à celle déjà observée sur des deniers émis dans cette ville ${ }^{28}$. Bien que

26. A. Morel-Fatio, dans A. Chabouillet, op . cit., n. 7, p. 39, cat. 160-161, pl. IX.

27. Un aperçu de plusieurs de ces représentations dans, J. LAFAURIE, Les animaux dans la numismatique mérovingienne, dans Le bestiaire des monnaies, des sceaux et des médailles, catalogue d'exposition, Hôtel de la monnaie, Paris, juin-septembre 1974, Paris, 1974, pl. I, cat. 2-3, pl. II, cat. 8-16.

28. Voir notamment le droit de BELFORT 559. 
proches dans leur représentation du droit, les deniers de Reims (?) avec au droit $\mathrm{RM}$, rétrograde ou non, surmonté d'un tilde dans un champ de points, ne nous semblent pas soutenir la comparaison ${ }^{29}$. En revanche, des analogies de style se remarquent dans le traitement des oiseaux figurant sur le denier de Rodez, particulièrement la gravure des ailes et des pattes, avec un denier au cavalier donné à la cité de Clermont et conservé au musée des Beaux-Arts de Lyon ${ }^{30}$ (figure 7). Surtout, un denier de même type découvert à Ins en Suisse (canton de Berne $)^{31}$ porte au droit un monogramme AR de gravure rétrograde en tout point similaire à celui de l'exemplaire du trésor de Rodez, mais sans la barre verticale située à droite du A que nous avons déjà signalée (figure 8). Malgré ce dernier détail, la convergence de ces spécificités stylistiques et iconographiques nous semble justifier l'attribution de ce denier à la cité clermontoise.

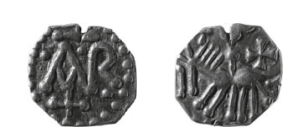

Figure 7 - Denier de Clermont.

(Musée des Beaux-Arts de Lyon, cat. 191).

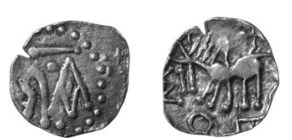

Figure 8 - Denier de Clermont trouvé à Ins.

(Service archéologique du canton de Berne, Inv. 135.0003)

Enfin, si l'on ne peut totalement exclure de lire NAR rétrograde sur le monogramme du denier de Rodez et orienter l'identification de son lieu d'émission vers Narbonne (Aude), l'indigence des frappes d'argent de cet atelier face à celles de Clermont ne plaide pas en faveur de cette hypothèse ${ }^{32}$.

29. Denier avec RM : BeLFoRT $1870=3786$, Prou 2851, MEC 605 ; denier avec RM rétrograde : BELFORT 3789 v., Prou 2852, MEC 604.

30. J. LAFAurie, op. cit., n. 4, p. 42-43, cat. 191 (BELFort 559 et 411).

31. J. LAFAURIE, Un denier mérovingien d'Arvernus trouvé à Anet / Ins, canton de Berne (Suisse), Archäologie im Kanton Bern 2. Fundberichte und Aufsätze, band. 2B, 1992, p. 413-417 ; Quelques trouvailles de monnaies mérovingiennes arvernes, BSFN, 50, 1995, p. 1048-1051.

32. Belfort 3173, Prou 2440. Deux exemplaires dans le trésor de Nice-Cimiez : A. MorelFatio, dans A. Chaboulllet, op. cit., n. 7, p. 25, cat. 106, pl. VII, nº 97. 


\section{Région de Marseille (Bouches-du-Rhône) ?}

Anépigraphe ; tête à g., avec une croix cantonnée de quatre globules devant le portrait. Anépigraphe ; croix grecque, supportant un oméga, accostée du chiffre VII. Poids 1,11 g ; diam. 12-11 mm ; Belfort 3053-3054 ; Prou - ; Depeyrot 4, p. 154.

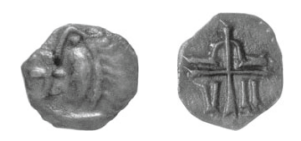

Plusieurs exemplaires de ce type monétaire sont inventoriés dans les trésors de Saint-Pierre-les-Étieux ${ }^{33}$, Nohanent ${ }^{34}$ et surtout de Nice-Cimiez ${ }^{35}$. A. Morel-Fatio proposait de lire au revers le monogramme MASS, quelque peu altéré, pour justifier la frappe de cette série à Massalia / Marseille. Si cette interprétation reste pour le moins conjecturale, c'est surtout la découverte de plusieurs deniers de ce type dans le trésor de Nice-Cimiez qui justifie leur attribution à cette ville ou plus vraisemblablement à un atelier proche, plutôt qu'à Mogunciacum (Mayence, Allemagne) comme l'avançaient anciennement B. Fillon puis A. de Belfort ${ }^{36}$. La découverte de ce nouvel exemplaire à Rodez abonderait, une nouvelle fois, en faveur de cette hypothèse méridionale ${ }^{37}$.

\section{Parisius, Paris (Paris)}

Attribuable à l'évêque Ragnecapdus ?, vers 740-750 ?

...ARISU... ; buste à d., la tête ceinte d'un important diadème se terminant par deux boucles pointées. Légende avec $\mathrm{S}$ couchée.

Anépigraphe ; croix ancrée cantonnée d'une perle aux 1,2 et sous le pendant du $4^{\mathrm{e}}$ quartier ; au 3 , accosté à la hampe, un croissant formant un $\mathrm{R}$ rétrograde, initiale de l'évêque.

Poids 0,83 g ; diam. 11 mm ; Belfort - ; Prou 757, même coin de droit ; Depeyrot 15.

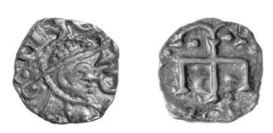

33. J. LafaURIE, op. cit., n. 8, p. 175, cat. 74-76, pl. XVI.

34. Ibid., p. 215, cat. 11-14, pl. XXI.

35. A. Morel-Fatio, dans A. Chaboulllet, op. cit., n. 7, p. 26-28, cat. 107, pl. VI-VII, $\mathrm{n}^{\text {os }} 106-118$.

36. Cette proposition est suivie notamment suivie par Ph. GrIERSOn \& M. BLACKBURN, Medieval European Coinage, 1, The Early Middle Ages (5th-10th Centuries), Cambridge, 1986-1991, p. 149.

37. Un exemplaire de ce type, mais qui ne figure pas dans l'ouvrage de LAFAurie - PILETLemière, est aussi signalé à Saint-Bauzille-de-Montmel (Hérault). Voir M. Feugère, G. DepeYrot, M.-L. Berdeaux-Le Brazidec, M. Bompaire, Catalogue du musée de Montagnac, I, Les monnaies antiques, médiévales et modernes (aquisitions 1987-1998), Montagnac, 2003, p. 65, fig. 57. 
Cette attribution repose sur le dernier classement des émissions monétaires épiscopales de Paris proposé par J. Lafaurie, complété de plusieurs analyses par neutrons rapides de J.-N. Barrandon ${ }^{38}$. L'auteur propose dans cet article d'identifier les différents évêques en charge de la fin du $\mathrm{VII}^{\mathrm{e}}$ siècle jusqu'au début du VIII ${ }^{\mathrm{e}}$ siècle sur les nombreux deniers épiscopaux émis par la ville grâce aux différents et initiales figurant dans le champ des revers. Selon ce classement, ce denier serait frappé au nom de l'évêque Ragnecapdus, dont l'épiscopat pourrait se situer vers $744-750^{39}$. Cette date reste incertaine car on ne sait que peu de choses sur ce personnage, sinon qu'il assuma ses fonctions après l'évêque Ratbertus, qui officiait peut-être aussi à Rouen en 744, et avant l'évêque Madalbertus qui détenait l'épiscopat avant $757^{40}$. L'attribution de ce denier à ce prélat repose sur la présence d'un croissant qui accoste le côté gauche de la hampe de la croix du revers pour former la lettre R, initiale de son nom. Ses deniers se distingueraient de ceux de son prédécesseur Ratbertus dont la même lettre initiale serait représentée cette fois «par l'extrémité des arcs de cercle du sommet de la croix, terminés par un trait horizontal qui leur donne la forme d'un $\mathrm{R}^{41}$. L'exemplaire du trésor de Rodez est, semble-t-il, de même coin de droit que celui conservé au Cabinet des Médailles (Prou 757) qui appartenait au trésor de Nice-Cimiez ${ }^{42}$ (figure 11).

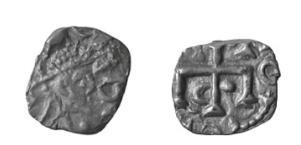

Figure 11 - Denier Prou 757 (Paris, BnF).

Bien que plusieurs différences soient à noter entre ces deux monnaies, les nombreuses similitudes qui les unissent laissent penser qu'il s'agit de la même matrice qui a été regravée puis réutilisée. Plusieurs cas similaires ont déjà été relevés sur des tremisses mérovingiens ${ }^{43}$.

38. J. Lafaurie, Monnaies épiscopales de Paris à l'époque mérovingienne, Cahiers de la Rotonde, 20, 1998, p. 61-99.

39. Ibid., p. 74.

40. Sur ces questions de chronologie, en plus des sources citées par J. Lafaurie, voir D.-F. IsEL, Prosopographie des personnages mentionnés dans les textes pour l'époque de Pépin le Bref et de son frère Carloman (741-768), $4^{\mathrm{e}}$ éd., s.1., 2006.

41. Nous reprenons ici les termes exacts employés par J. Lafaurie pour distinguer ces deux monnayages et justifier sa typologie. En revanche, l'auteur ne précise pas son choix pour l'un ou l'autre de ces évêques pour chacune de ces émissions.

42. Ainsi que le denier BelFort 6321, Prou 758. Ces deux deniers ont été acquis avant le don Morel-Fatio au Cabinet des Médailles.

43. Plusieurs exemples dans, M. DHÉNIN, La regravure des coins monétaires, dans T. HACKENS, R. WeIller (éd.), Actes du $9^{e}$ Congrès International de Numismatique, Berne, Septembre 1979, II, Louvain-la-Neuve, Luxembourg, 1982, p. 1039-1046, pl. 120-122. 


\section{Parisius, Paris (Paris)}

Attribuable à l'évêque Deodefridus ?, vers 750-756 ?

...ARI...V... ; buste à d., ceint d'un important diadème se terminant par deux boucles. Anépigraphe ; croix ancrée cantonnée d'une perle au 1 (?) et 2, entourée d'un grènetis coupé par des annelets. Croissant vertical appuyé sur le coté droit de la hampe de la croix formant un D, initiale de l'évêque. Au-dessus, se devine une croix.

Poids 1,01 g ; diam. 12-11 mm ; Belfort - ; Prou - ; Depeyrot -.

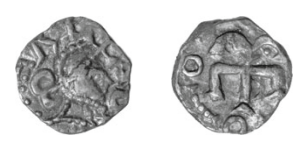

L'identification de ce second denier épiscopal de Paris s'est révélée plus délicate que pour le précédent exemplaire. Le croissant accostant la hampe de la croix du revers se situe cette fois du côté droit et peut aussi former un $\mathrm{R}$ mais d'un type qui n'a été reconnu sur aucun des deniers parisiens étudié par J. Lafaurie. Cette lettre pourrait alors correspondre à l'initiale de Ratbertus, voire de Ragnecapdus et compléter les émissions monétaires déjà identifiées pour l'un de ces deux évêques. Ce croissant pourrait aussi s'interpréter comme un B, dont la partie inférieure aurait été tronquée, et renvoyer à l'initiale de l'évêque Berneharius dont l'épiscopat se situe vers 720-730 (figure 13). Cette explication n'est pourtant pas satisfaisante au regard des autres deniers connus pour ce prélat dont le B gravé au revers est parfaitement lisible ${ }^{44}$.

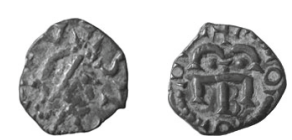

Figure 13 - Denier Prou 753 (Paris, BnF).

Reste une dernière proposition : le croissant accosté au jambage, que l'on peut tenter de déchiffrer comme un possible $\mathrm{R}$ ou encore un possible $\mathrm{B}$, peut tout aussi bien, et surtout plus simplement, s'interpréter comme un D, initiale de l'évêque Déodefridus. Aucune monnaie n'est encore attribuée à ce prélat, dernier connu sur la liste des évêques mérovingiens de Paris et qui n'apparaît que dans une brève mention sous le règne de Pépin le Bref pour l'année 756.

44. Voir notamment les exemplaires 10 (Prou 753), 11 (Prou 755) et 12 (Prou 751) illustrés dans J. LAFAURIE, op. cit., n. 38, p. 88. 
C'est en effet Déodefridus qui signe l'acte de confirmation des donations de Chrodegard, évêque de Metz, au monastère de Gorze le 18 mai de cette année ou sinon la suivante ${ }^{45}$. Appartenait-il à l'épiscopat mérovingien, tout comme son prédécesseur Madalbertus pour lequel les dates sont aussi imprécises? L'hypothèse reste malgré tout envisageable et si l'on accorde crédit à la chronologie proposée par J. Lafaurie et à cette attribution, le petit dépôt funéraire de Rodez pourrait constituer le plus récent trésor mérovingien actuellement connu dont la période d'enfouissement pourrait se réduire aux années 750-760, soit dans les toutes premières années du pouvoir carolingien.

\section{Atelier indéterminé}

Anépigraphe ; tête informe à d. ou monogramme ?

Anépigraphe ; croix cantonnée des lettres D au 1, C au 2, A au 3 et D au 4.

Poids 0,51 g ; diam. 11-10 mm ; Belfort 5721 ; Prou 2785, même coin de droit ;

Depeyrot 2, p. 167.

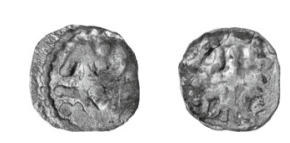

L'aspect émoussé de la monnaie ne semble pas la conséquence d'une longue circulation mais plutôt de l'emploi de coins usés pour sa frappe. Malgré son aspect usé, ce denier est bien issu du même coin de droit que l'exemplaire conservé au Cabinet des Médailles (Prou 2785) (figure 15), mais d'une masse beaucoup plus faible (cf. infra). La frappe, mieux centrée au revers, permet de confirmer en partie la lecture de l'exemplaire du Cabinet des Médailles de Paris : le D du premier canton apparaît complet sur le denier de Rodez, le C du deuxième canton pourrait aussi être lu comme un A mais sans barre.

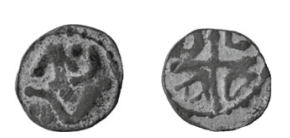

Figure 15 - Denier Prou 2785 (Paris, BnF).

L'interprétation iconographique du droit reste quant à elle tout aussi difficile à cerner. Ce denier est le plus léger du trésor du Rodez. Le trésor de NiceCimiez contenait deux exemplaires de ce type ${ }^{46}$.

45. En 756 selon J. Lafaurie, op. cit., n. 38, p. 74 ; en 757 selon D.-F. Isel, op. cit., n. 40. 46. A. Morel-Fatio, dans A. Chabouillet, op. cit., n. 7, p. 55, cat. 278. 


\section{Atelier indéterminé}

...MVA... ; croix posée sur un oméga retourné, le tout dans un diadème aux extrémités recourbées.

$+\ldots$; monogramme non identifié ponctué de globules dans le champ.

Poids $1,18 \mathrm{~g}$; diam. $14 \mathrm{~mm}$; à rapprocher notamment de Belfort 6682 ; Prou 2767 ; Depeyrot 4, p. 169.

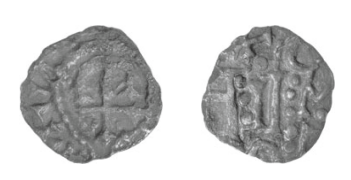

Ce denier n'a pas d'équivalent dans toute la documentation que nous avons pu consulter. Tout au plus peut-on le comparer stylistiquement à quelques frappes de lecture incertaine dont notamment le type Belfort 6682 (figure 17).

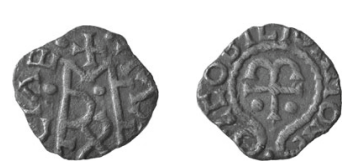

Figure 17 - Denier Prou 2767 (Paris, BnF).

Les principales caractéristiques du droit, avec la croix dans un diadème ceint d'une légende, s'apparentent à cet exemplaire, tout comme le revers avec une croix initiale surmontant un monogramme ou un agencement de lettres indéterminé.

\section{Atelier indéterminé}

+IDNH[ ]; lettres I B avec B pointé.

[ ]; protubérance (?) entourée d'un grènetis.

Poids 1,20 g ; diam. 10 mm ; Belfort 6686 ; Prou 2809 ; Depeyrot 21, p. 171. 
Comme pour l'exemplaire $\mathrm{n}^{\circ} 7$, ce denier est indéterminé mais pas inconnu de Belfort, Prou et Depeyrot qui le recensent tous trois dans leurs corpus. Son état est identique à celui conservé au cabinet des Médailles de Paris avec la même face lisible, bien que plus usée, l'autre étant totalement fruste, mais portant aussi les vestiges d'un grènetis. La consultation d'un troisième exemplaire conservé dans une collection privée a permis de constater les mêmes caractéristiques. Si ce denier est peut-être un nouveau cas d'utilisation d'un coin bouché, on ne peut non plus exclure qu'il s'agisse d'une volonté délibérée de procéder à un tel type de frappe uniface. Malgré quelques différences visibles, il se pourrait que le même coin de droit ait été utilisé pour la frappe de ces deux deniers mais après avoir été regravé comme dans le cas du denier de Paris au nom de Ragnecapdus (cat. 5).

La légende à peine visible qui entoure les lettres I B semble en effet très proche de celle de Prou 2809 (figure 19), mais on relèvera la forme différente de la partie basse du B qui aurait pu faire l'objet d'une retouche. Un autre denier indéterminé assez proche de celui-ci existe aussi avec I B au revers mais complété d'un B rétrograde plus petit, placé devant le I, sans légende autour. Le revers associé est aussi fruste mais ponctué d'un unique annelet dans le cœur du champ, sans grènetis apparent. Un exemplaire de ce type figure notamment dans le trésor de Plassac ${ }^{47}$.

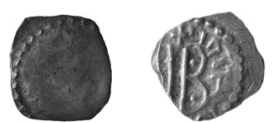

Figure 19 - Denier Prou 2809 (Paris, BnF).

\section{Examen pondéral}

La masse totale du trésor de Rodez s'établit à $8,53 \mathrm{~g}$ soit une masse moyenne de $0,947 \mathrm{~g}$. Ce chiffre est très faible si on le compare à la masse théorique de $1,35 \mathrm{~g}$ environ de ces deniers : il n'en représente que les deux tiers. Trois deniers dépassent franchement la masse d' $1 \mathrm{~g}$ (cat. $4,1,11 \mathrm{~g}$; cat. 8,1,18 g et cat. 9, $1,20 \mathrm{~g}$ ) mais sans jamais atteindre le seuil théorique ; deux autres flirtent avec ce seuil d'1 g (cat. 3, 0,99 g et cat. 6,1,01 g). La masse la plus faible est de $0,51 \mathrm{~g}$ pour le cat. $7^{48}$.

47. J. Lafaurie, op. cit., n. 8, p. 207, cat. 154, pl. XX ; Belfort 5681 ; Prou 2835.

48. Le denier Prou 2785, de même coin de droit, pèse $0,90 \mathrm{~g}$, soit presque le double! De même, le denier Prou 757 issu du même coin de droit que le denier $\mathrm{n}^{\circ} 5$ du trésor pèse $1,06 \mathrm{~g}$ contre $0,83 \mathrm{~g}$ pour l'exemplaire du trésor de Rodez. 
Tableau 1 - Masse des deniers mérovingiens dans les principaux trésors découverts en France (D'après J. LAFAURIE, op. cit., n. 8, p. 145).

\begin{tabular}{|c|c|c|c|c|c|c|c|c|c|}
\hline & & \multirow{2}{*}{ Rodez } & \multirow{2}{*}{ St-Pierre } & \multirow{2}{*}{ Plassac } & \multirow{2}{*}{ Bais } & \multirow{2}{*}{ Savonnières } & \multicolumn{2}{|c|}{ Nice-Cimiez } & \multirow[t]{2}{*}{ Total } \\
\hline & & & & & & & Austrasie & Provence & \\
\hline 0,5 & 0,6 & 1 & - & - & - & - & - & - & 1 \\
\hline 0,61 & 0,65 & - & - & 1 & - & - & 3 & - & 4 \\
\hline 0,66 & 0,7 & - & - & - & 1 & - & 2 & - & 3 \\
\hline 0,71 & 0,75 & - & - & - & - & - & 3 & - & 3 \\
\hline 0,76 & 0,8 & 1 & - & 1 & 2 & - & 2 & 1 & 7 \\
\hline 0,81 & 0,85 & 1 & 2 & - & 6 & - & 8 & 4 & 21 \\
\hline 0,86 & 0,9 & 1 & - & - & 9 & 1 & 11 & 4 & 26 \\
\hline 0,91 & 0,95 & - & 2 & 4 & 18 & 6 & 12 & 7 & 49 \\
\hline 0,96 & 1 & 1 & 1 & 6 & 22 & 3 & 16 & 11 & 61 \\
\hline 1,01 & 1,05 & 1 & 5 & 5 & 25 & 2 & 28 & 27 & 92 \\
\hline 1,06 & 1,1 & - & 5 & 11 & 36 & 3 & 27 & 46 & 128 \\
\hline 1,11 & 1,15 & 1 & 6 & 9 & 49 & 2 & 41 & 53 & 161 \\
\hline 1,16 & 1,2 & 2 & 7 & 11 & 49 & 2 & 31 & 32 & 134 \\
\hline 1,21 & 1,25 & - & 22 & 15 & 43 & - & 30 & 13 & 123 \\
\hline 1,26 & 1,3 & - & 11 & 10 & 53 & - & 27 & 10 & 111 \\
\hline 1,31 & 1,35 & - & 7 & 6 & 28 & - & 14 & 6 & 61 \\
\hline 1,36 & 1,4 & - & 3 & 8 & 10 & - & 10 & - & 31 \\
\hline 1,41 & 1,45 & - & 1 & - & 3 & - & 5 & 1 & 10 \\
\hline 1,46 & 1,5 & - & - & - & - & - & 1 & 1 & 2 \\
\hline \multicolumn{2}{|c|}{$1,50 \mathrm{et}+$} & - & - & - & - & - & 1 & - & 1 \\
\hline \multicolumn{2}{|c|}{ Total } & 9 & 72 & 87 & 354 & 19 & 272 & 216 & 1029 \\
\hline
\end{tabular}

Si l'on compare ces chiffres avec ceux d'autres trésors, plus particulièrement, ceux de Saint-Pierre-les-Étieux, Plassac, Bais, Savonnières et de Nice-Cimiez, ils correspondent à la moyenne la plus basse. La quantité sur laquelle nous raisonnons est faible mais cette spécificité des deniers de Rodez s'accorderait, sur ce plan aussi, avec leur interprétation comme un ensemble de frappes tardives.

\section{Datation du trésor}

Le jalon chronologique principal dont nous disposons pour dater ce petit dépôt est la présence de deux deniers épiscopaux frappés à Paris. Selon le classement proposé par J. Lafaurie, à l'appui de l'étude des évêques de la ville par D. Dubois, le premier de ces deniers serait attribuable à Ragnecapdus (vers 744-750). Le second, qui semble inédit, pourrait avoir été émis, d'après la méthode utilisée dans les travaux précédemment cités, au nom de l'évêque Deodefridus (vers 740-756), dont aucune monnaie ne nous est encore parvenue et dont on sait seulement qu'il exerçait une charge épiscopale en 756/757. Sans que l'on puisse la cerner plus précisément, l'attribution de ce denier viendrait alors confirmer cette charge épiscopale avant 751, date butoir proposée à ce jour de l'arrêt des 
frappes d'argent mérovingiennes. Dès lors, l'enfouissement du dépôt funéraire de Rodez pourrait être fixé à une période sensiblement contemporaine de celui du trésor de Nice-Cimiez, dans la décennie 750-760. Les motivations en sont pourtant différentes puisqu'il ne s'agissait pas dans le cas de Rodez de préserver un pécule mais au contraire de le confier comme viatique au défunt. Sa valeur est d'ailleurs très faible puisqu'elle ne correspond même pas à un tremissis si l'on se reporte au ratio presque constant de 12 monnaies d'argent pour une monnaie d'or.

La convergence de certains indices numismatiques est aussi à prendre en compte pour la datation de cet ensemble. Ainsi, on relèvera la présence de trois monnaies frappées avec des coins particulièrement usés (cat. 1, 7 et 9) ainsi qu'une autre, au moins, (cat. 5), avec un coin regravé. De tels exemples ont déjà été relevés dans d'autres trésors mais sans jamais atteindre la proportion d'un tiers de l'ensemble comme c'est le cas pour le dépôt de Rodez. Cette spécificité est peut-être à mettre en relation avec les dernières productions de ces monnayages d'argent. Elle ne serait d'ailleurs pas contredite par les analyses élémentaires qui montrent une grande hétérogénéité dans la composition de ces quelques pièces.

Enfin, le dernier indice dont nous disposons est la chronologie générale des tombes fouillées sur le site des Jacobins de Rodez. Elle se situe entre le $\mathrm{VII}^{\mathrm{e}}$ et le $\mathrm{VIII}^{\mathrm{e}}$ siècle. Cette datation est notamment confirmée par une analyse radiocarbone réalisée sur l'individu de la sépulture 7 au côté duquel étaient déposées les monnaies. Ces analyses proposent deux intervalles chronologiques : l'un compris entre les années 660 et 785 à $83,1 \%$ de fiabilité et le second entre les années 785 et 865 à $13,5 \%$ de fiabilité ${ }^{49}$. Cette première datation est pleinement confirmée par les monnaies recueillies dans la tombe, puisque les deniers apparaissent vers 675 et cessent d'être émis aux environs de 750. L'intervalle chronologique durant lequel ces monnaies sont frappées s'intègre donc pleinement au sein de cette période. Une datation dans les dernières années du pouvoir mérovingien n'est donc pas incompatible. L'abandon de ce dépôt pourrait d'ailleurs tout à fait s'envisager dans les années 755-760, date à laquelle Pépin le Bref fixe de nouvelles normes, consignées dans le capitulaire de Ver en 755 , pour la fabrication des deniers. Si la masse des nouvelles monnaies d'argent diffère peu, leur module, plus large (environ $15 \mathrm{~mm}$ ), les distingue nettement des précédentes. L'application de cette ordonnance a pu provoquer le retrait ou le décri des espèces antérieures, les rendant obsolètes dans la circulation, et contribuer ainsi à ce qu'elles soient déposées comme viatique pour accompagner la défunte.

49. Analyses effectuées par le laboratoire Archéolabs. Date ${ }^{14} \mathrm{C}$ calibrée : $660 \mathrm{cal}$ AD - 865 cal AD (courbe de calibration «IntCal98»). 


\section{Le trésor de Cimiez et le trésor de Rodez : un problème de chronologie}

Cette proposition ne saurait pourtant être considérée comme idéale, car elle se heurte en premier lieu à la datation proposée pour l'enfouissement du trésor de Nice-Cimiez. Tous les numismates ne s'accordent pas sur la date de cet événement que $\mathrm{Ph}$. Grierson et M. Blackburn cernent vers $720^{50}$, J. Lafaurie autour de $740^{51}$ et $\mathrm{G}$. Depeyrot aux environ de $745^{52}$. Si le petit dépôt funéraire de Rodez et le volumineux trésor provençal se situent tous deux aux antipodes sur un plan quantitatif, ils n'en comportent pas moins quelques points communs. Plus particulièrement, cinq deniers du trésor de Rodez figurent dans celui de Nice-Cimiez, à savoir ceux de Brioude (cat. 1), de Brioux-sur-Boutonne (cat. 2), de la région de Marseille (cat. 4), de Paris (cat. 5) ainsi qu'un exemplaire indéterminé (cat. 7). Surtout, deux de ces deniers (cat. 5 et 7) sont issus des mêmes coins de droit que deux exemplaires appartenant au trésor niçois et actuellement conservés au Cabinet des Médailles de Paris. L'abandon du petit dépôt ruthène ne peut donc se concevoir sans aborder le cas «Nice-Cimiez». Il faut convenir que, sur ce point, la position de nos illustres collègues britanniques est sans conteste la plus raisonnée, se basant sur la quantité impressionnante de deniers au nom du patrice Nemfidius (plus de la moitié du trésor) dont la charge est assurée vers 700. Il est donc difficile au regard de cette masse monétaire d'envisager un enfouissement du trésor trois décennies plus tard.

Néanmoins, on objectera deux éléments sur ce point. Tout d'abord, la possibilité d'une thésaurisation en deux temps qui aura permis la constitution d'un premier lot particulièrement conséquent auquel plusieurs centaines de monnaies seront ajoutées ensuite. S'il ne s'agit là que d'une hypothèse, l'examen des découvertes de sites de la région marseillaise s'avère en revanche plus probant, notamment celles de Glanum / Saint-Rémy-de-Provence. Ainsi, les trouvailles isolées de deniers au nom de Nemfidius ${ }^{53}$ recueillies sur le site s'avèrent, et de loin, les plus abondantes avec treize exemplaires, contre deux seulement pour le patrice Ansedert ${ }^{54}$ et une seule pour Antenor ${ }^{55}$. Malgré la faiblesse de l'échantillon à disposition, les rapports entre ces monnayages sont assez proches de ceux relevés dans le trésor de Cimiez. Dans le cas des trouvailles isolées de Glanum, les déséquilibres quantitatifs existant entre les monnayages aux noms des trois patrices ne sont pas nécessairement liés à un pic d'occupation du site

50. Ph. Grierson, M. Blackburn, op. cit., n. 36, p. 142-143.

51. J. Lafaurie, op. cit., n. 8, p. 107 et J. Lafaurie, J. Pilet-Lemière, op. cit., n. 5, p. 52.

52. G. DePEYRot, op. cit., n. 20, p. 15.

53. J. Lafaurie, J. PIlEt-Lemière, op. cit., n. 5, p. 81, 87-88 recensent 15 trouvailles de sites, dont 13 à Glanum - Saint-Rémy-de-Provence, pour ce seul patrice. L'une d'entre elles est considérée comme douteuse et provient peut-être... du trésor de Cimiez.

54. Deux autres deniers d'Ansedert sont recensés à Eyguières et Fréjus. Ibid., p. 78 et 325.

55. Un autre denier d'Antenor est inventorié, sans plus de précision, «en Provence», Ibid., p. 90. 
mais plus sûrement représentatifs des volumes monétaires produits par chacun d'eux. À ce titre la quantité de coins gravés au nom de Nemfidius en témoigne. Si les tentatives pour identifier d'autres personnages postérieurs à ce patrice n'ont pas manqué, aucune n'a vraiment remporté l'unanimité, loin s'en faut. Seul actuellement le classement du monnayage des évêques de Paris publié par J. Lafaurie permet de bouleverser la chronologie proposée pour le trésor de Cimiez et repousser sa date à partir de 740/5. C'est sur la base de ce travail que nous avançons aussi celle de l'abandon du viatique découvert dans la sépulture de Rodez. Si certaines attributions sont susceptibles d'être modifiées au sein de ce classement entre les évêques Ratbertus, Ragnecapdus, Madalbertus et Déodefridus, c'est donc plus largement, et plus prudemment aussi, entre 730 et 760 que nous proposerons de dater l'enfouissement du petit trésor ruthène en penchant pour les années 750-760.

\section{Analyses de composition}

Compte-tenu de la rareté de ce type de trouvaille et au regard du faible nombre des exemplaires qui la composent, il paraissait indispensable de pouvoir procéder à des analyses de composition des alliages constituant ces deniers. Ce fut chose faite grâce à $\mathrm{M}$. Bompaire qui a pu intégrer cette intervention dans le cadre d'un programme de recherche sur l'argent carolingien dans lequel s'était investi le Centre Ernest Babelon (IRAMAT - UMR 5060, CNRS, Université d'Orléans), et plus particulièrement dans celui d'une thèse récemment soutenue et consacrée à ce numéraire ${ }^{56}$.

Deux méthodes d'analyse élémentaire complémentaires ont été mises en œuvre pour la caractérisation des alliages constituant les pièces du trésor de Rodez : la spectrométrie de masse couplée à un plasma inductif avec prélèvement par ablation laser (LA-ICP-MS), et l'activation aux neutrons rapides de cyclotron (ANRC). L'ANRC est une méthode d'analyse élémentaire globale qui fournit une valeur moyenne déterminée sur l'ensemble de l'objet pour chaque élément détecté. La méthode LA-ICP-MS, récemment appliquée avec succès aux monnaies anciennes à base d'argent, permet d'analyser des micro-prélèvements invisibles à l'œil nu effectués à l'aide d'un laser. Ce mode d'ablation particulier permet de représenter graphiquement l'évolution de la composition de l'alliage selon la profondeur, et donc de visualiser l'enrichissement de la surface en argent qui affecte fréquemment les alliages anciens à base d'argent. Cette représentation permet de s'affranchir de cette couche de surface perturbée pour analyser l'alliage sain dont la composition correspond à celle de la monnaie au moment

56. G. SARAH, Caractérisation de la composition et de la structure des alliages argent-cuivre par ICP-MS avec prélèvement par ablation laser. Application au monnayage carolingien, Thèse de l'Université d'Orléans, 2008, 2 vol. 
de sa frappe ${ }^{57}$. Cette méthode permet en outre de doser un plus grand nombre d'éléments que l'ANRC, et présente des limites de détection très basses. Pour la méthode LA-ICP-MS, trois analyses ont été effectuées en différents endroits de chaque monnaie afin de s'assurer de l'homogénéité de l'alliage.

Les analyses réalisées par LA-ICP-MS à partir de micro-prélèvements ont permis d'obtenir des profils de concentration stables et reproductibles pour les monnaies 1 à 5 et 7 du trésor mérovingien de Rodez. Les compositions déterminées grâce à cette méthode sont, de plus, en bon accord avec les analyses par ANRC. Ces deux observations suggèrent une bonne conservation des alliages monétaires durant leur enfouissement.

Pour les monnaies 8 et 9 , les profils de concentration de l'argent se sont révélés plus erratiques que pour les exemplaires cités précédemment. Leur superposition permet toutefois de proposer des compositions pour ces deniers avec une bonne confiance.

Reste la monnaie 6 pour laquelle l'approche ponctuelle par LA-ICP-MS s'est avérée inadaptée : aucun des profils de concentration obtenus pour l'argent par cette méthode ne forme de palier de stabilité permettant de déterminer une composition. L'analyse globale par ANRC était donc indispensable afin de proposer une composition de cet échantillon. D'une manière générale, les autres monnayages analysés jusqu'à maintenant par LA-ICP-MS, et en particulier les deniers carolingiens, n'ont pas révélé de telles hétérogénéités et les résultats obtenus étaient fiables dans la majorité des cas.

Les titres d'argent des neuf deniers mérovingiens découverts à Rodez sont compris entre $64 \%$ et $92 \%$ (tableau 2). Trois d'entre eux contiennent de $90 \%$ à $92 \%$ d'argent (4, 7 et 9$)$, trois autres de $80 \%$ à $90 \%$ (1, 3 et 5), et les trois derniers de $64 \%$ à $80 \%$ (2, 6 et 8$)$. Des monnaies constituées d'alliages de puretés diverses sont donc rassemblées au sein de ce trésor.

Par le passé, deux principales publications ont présenté des résultats d'analyses élémentaires de deniers d'argent mérovingiens : celle de D.M. Metcalf d'une part, qui regroupe cent résultats obtenus par une méthode d'analyse de surface sur des deniers mérovingiens ainsi que sur quelques sceattas frisons ${ }^{58}$; celle de J. Lafaurie d'autre part, spécifique aux émissions des évêques de Paris, qui rassemble une trentaine de compositions de telles pièces obtenues par une

57. Sur l'application de cette méthode d'analyse spécifiquement aux monnaies d'argent voir G. SARAH, B. GratuZe et J.-N. BARRAndon, Application of laser ablation inductively coupled plasma mass spectrometry (LA-ICP-MS) for the investigation of ancient silver coins, Journal of Analytical Atomic Spectrometry, 22, 2007, p. 1163-1167. Voir également G. SARAH, M. BOMPAIRE, M. McCormick, A. Rovelli, C. Guerrot, Analyses élémentaires de monnaies de Charlemagne et Louis le Pieux du Cabinet des Médailles : 1'Italie carolingienne et Venise, $R N$, 2008, p. 355-406, en particulier p. 361-364.

58. D.M. MetCALF, Interpreting the alloy of the Merovingian silver coinage, dans C.N.L. BROOKE, B.H.I.H. Stewart, J.G. Pollard, T.R. Volk (éd.), Studies in Numismatic Method presented to Philip Grierson, Cambridge University Press, Cambridge, 1983, p. 113-126. 
Tableau 2 - Composition élémentaire des deniers du trésor de Rodez. Analyses par LA-ICP-MS sauf Rodez 6 par ANRC. Ag, Cu, Pb, Au, Zn et Sn en \%, autres éléments en ppm (10000 ppm = $1 \%)$. Analyses G. Sarah, Centre Ernest Babelon, Orléans.

\begin{tabular}{|c|c|c|c|c|c|c|c|c|c|}
\cline { 2 - 9 } \multicolumn{1}{c|}{} & Rodez 1 & Rodez 2 & Rodez 3 & Rodez 4 & Rodez 5 & Rodez 6 & Rodez 7 & Rodez 8 & Rodez 9 \\
\hline $\mathbf{A g}$ & $82,7 \%$ & $64,7 \%$ & $88,2 \%$ & $91,4 \%$ & $87,0 \%$ & $71,8 \%$ & $90,2 \%$ & $77,2 \%$ & $90,8 \%$ \\
$\mathbf{C u}$ & $13,1 \%$ & $27,6 \%$ & $7,3 \%$ & $5,3 \%$ & $10,7 \%$ & $22,4 \%$ & $4,1 \%$ & $14,0 \%$ & $6,0 \%$ \\
\hline $\mathbf{P b}$ & $0,91 \%$ & $1,37 \%$ & $1,63 \%$ & $1,04 \%$ & $0,59 \%$ & $1,62 \%$ & $0,59 \%$ & $2,46 \%$ & $0,33 \%$ \\
$\mathbf{A u}$ & $1,39 \%$ & $0,98 \%$ & $0,91 \%$ & $1,11 \%$ & $1,63 \%$ & $0,85 \%$ & $4,47 \%$ & $0,54 \%$ & $2,75 \%$ \\
$\mathbf{Z n}$ & $0,20 \%$ & $0,69 \%$ & $0,37 \%$ & $0,08 \%$ & $0,02 \%$ & $1,58 \%$ & $0,03 \%$ & $0,62 \%$ & $0,02 \%$ \\
Sn & $1,61 \%$ & $4,31 \%$ & $1,32 \%$ & $1,00 \%$ & $0,10 \%$ & $1,66 \%$ & $0,51 \%$ & $4,85 \%$ & $0,06 \%$ \\
\hline
\end{tabular}

\begin{tabular}{|c|c|c|c|c|c|c|c|c|c|}
\hline $\mathbf{N i}$ & 12 & 28 & 228 & 49 & 58 & $n d$ & 5,6 & 15 & 24 \\
$\mathbf{A s}$ & 262 & 1213 & 374 & 39 & 16 & $n d$ & 11 & 873 & 13 \\
$\mathbf{P d}$ & 2,3 & 3,0 & 4,0 & 3,5 & 4,2 & $n d$ & 3,8 & nd & 3,0 \\
$\mathbf{C d}$ & 0,61 & 0,8 & 1,4 & 0,13 & 0,14 & $n d$ & 1,3 & 2,2 & 2,9 \\
$\mathbf{S b}$ & 397 & 1010 & 421 & 55 & 62 & 299 & 22 & 948 & 34 \\
$\mathbf{T e}$ & 2,1 & 2,5 & 12 & 3,9 & 3,8 & $n d$ & 1,8 & 7,4 & 1,6 \\
$\mathbf{P t}$ & 2,4 & 2,0 & 5,8 & 2,8 & 3,6 & $n d$ & 0,91 & 2,1 & 3,9 \\
$\mathbf{B i}$ & 250 & 189 & 1065 & 266 & 160 & $n d$ & 148 & 685 & 221 \\
\hline $\mathbf{A u} / \mathbf{A g}$ & $1,68 \%$ & $1,51 \%$ & $1,04 \%$ & $1,21 \%$ & $1,87 \%$ & $1,18 \%$ & $4,96 \%$ & $0,70 \%$ & $3,03 \%$ \\
$\mathbf{Z n} / \mathbf{C u}$ & $1,51 \%$ & $2,48 \%$ & $5,02 \%$ & $1,43 \%$ & $0,19 \%$ & $7,04 \%$ & $0,68 \%$ & $4,42 \%$ & $0,29 \%$ \\
$\mathbf{S n} / \mathbf{C u}$ & $12,34 \%$ & $15,62 \%$ & $18,05 \%$ & $18,63 \%$ & $0,92 \%$ & $7,40 \%$ & $12,21 \%$ & $34,63 \%$ & $0,95 \%$ \\
\hline
\end{tabular}

méthode de caractérisation globale ${ }^{59}$. De telles contributions sont certes insuffisantes pour une compréhension globale de la frappe des deniers mérovingiens entre le dernier quart du $\mathrm{VII}^{\mathrm{e}}$ siècle et les années 750 , mais elles apportent des éléments de réflexion importants pour l'étude de la pureté des alliages monétaires d'argent de la fin de la période mérovingienne. Avant même d'envisager la comparaison des résultats obtenus pour les deniers du trésor de Rodez avec ceux présentés dans les deux publications précitées, les limitations d'une telle démarche doivent être exposées.

La première limitation tient à la différence de caractéristiques intrinsèques de la méthode analytique mise en œuvre dans chacune des trois études considérées. D.M. Metcalf a employé une méthode d'analyse de surface et, conscient des spécificités de structure des monnaies anciennes en argent, il s'est efforcé de s'affranchir du phénomène d'enrichissement en argent de la surface des pièces pour déterminer la composition la plus juste possible. J.-N. Barrandon a analysé en collaboration avec J. Lafaurie les deniers épiscopaux de Paris par ANRC, méthode nucléaire d'analyse globale. Enfin, nos résultats, comme cela a été exposé plus haut, ont été obtenus suite à la réalisation de profils de concentration ponctuels par LA-ICP-MS.

59. J. LAFAURIE, op. cit., n. 38. Voir en particulier les résultats présentés p. 84 et leur interprétation p. 82-85. 
Nous avons vu que la corrosion des alliages monétaires peut influencer leur structure interne ; l'approche ponctuelle par LA-ICP-MS peut alors s'avérer inappropriée, et la mise en œuvre d'une méthode d'analyse complémentaire dans notre étude a permis de s'affranchir de cette limitation, et donc de proposer des résultats fiables pour chacune des pièces du trésor de Rodez. L'analyse globale par ANRC appliquée aux monnaies d'argent permet d'obtenir des résultats justes et fiables dans la majorité des cas. Seule la combinaison de particularités géométriques et de composition des monnaies peut être la source d'une surestimation du titre d'argent lors de la mise en œuvre d'une telle méthode ${ }^{60}$. Les résultats obtenus par LA-ICP-MS doivent toutefois être privilégiés si les profils de concentration démontrent une structure homogène de l'alliage sous la couche de corrosion : cette approche permet en effet une meilleure confiance dans les résultats, ainsi qu'un dosage plus précis d'un plus grand nombre d'élémentstraces que les autres techniques. Les deniers mérovingiens épiscopaux de Paris ne présentent pas de caractéristiques de géométrie qui pourraient être la cause d'une surestimation du titre d'argent par ANRC, aussi il est envisageable de comparer nos résultats avec ceux présentés dans l'article de J. Lafaurie, pour les constituants majeurs comme pour les éléments mineurs indiqués dans cette publication. Les valeurs proposées par D.M. Metcalf sont en revanche à considérer avec davantage de précautions. En effet, malgré la mise en œuvre d'une procédure de nettoyage et d'analyse répétée pour s'affranchir d'une éventuelle couche de surface perturbée, des hétérogénéités importantes pour un même échantillon sont apparues. D.M. Metcalf rapporte les difficultés rencontrées lors de la caractérisation des pièces de son corpus de deniers mérovingiens ${ }^{61}$.

Les difficultés de classement et d'attribution de nombre de deniers mérovingiens constituent également une limitation importante pour l'interprétation de résultats analytiques et pour la comparaison des valeurs obtenues par différents analystes. Au sein du corpus de deniers frappés sous la responsabilité d'évêques de Paris rassemblé par J. Lafaurie, des incertitudes demeurent quant à l'attribution de chaque exemplaire considéré à un prélat connu ; les dates d'épiscopat de certains des évêques identifiés ou supposés sont, de plus, incertaines ou inconnues. L'étude du corpus monétaire de D. M. Metcalf, plus large et plus varié que celui

60. Les limitations liées à ces caractéristiques ne concernent par seulement l'ANRC mais toute méthode d'analyse globale appliquée à des monnaies d'argent. Voir G. SARAH, op. cit., n. 56, p. 24-37 et p. 160-163. Voir également J. Condamin, M. Picon, Changes suffered by coins in the course of time and the influence of these on the results of different methods of analysis, dans E.T. Hall, D.M. Metcalf (éd.), Methods of chemical and metallurgical investigation of ancient coinage, Londres, (RNS Special Publication, 8), 1972, p. 49-66.

61. D.M. Metcalf, op . cit., n. 58,p.113-114. En particulier p. $113:$ : Analysis on an abraded section on the edge of the coin should in theory discount surface effects, such as corrosion or surface enrichment, and should measure the unaltered alloy; in practice, however, it is a delicate and sometimes perhaps an impossible task, within the limitation of acceptable aesthetic damage, to obtain even an approximately correct result from these tiny coins, particularly when they are debased.». 
de J. Lafaurie, met en évidence les difficultés auxquelles est confronté le chercheur spécialiste des monnaies d'argent mérovingiennes. Et, contrairement au texte relatif aux deniers épiscopaux de Paris, celui de D.M. Metcalf ne présente pas de véritable étude numismatique mais seulement une interprétation très succincte des résultats analytiques. Le corpus de deniers mérovingiens constitué par le trésor de Rodez pose également de nombreuses questions de datation et d'attribution qui ont été développées plus haut. C'est donc sur ces bases, et avec la connaissance de leurs faiblesses, que peut s'effectuer la comparaison des résultats des analyses des deniers du trésor de Rodez, avec les pièces mérovingiennes caractérisées antérieurement. Un quatrième lot de monnaies a été intégré dans cette étude : il s'agit de quinze deniers de Marseille issus du trésor de NiceCimiez et conservés au Cabinet des Médailles de la Bibliothèque nationale de France. Ces analyses ont été réalisées par J. Poirier et J.-N. Barrandon par activation neutronique au cours de l'année 1982 à la demande de J. Lafaurie, et n'avaient pas été publiées. Ces résultats ont donc été intégrés à cette étude pour comparaison $^{62}$.

Tableau 3 - Teneurs en argent de quelques deniers du trésor de Nice-Cimiez conservés à la BnF. Résultats inédits de J. Poirier et J.-N. Barrandon, Orléans, 1982.

\begin{tabular}{|c|c|c|c|}
\hline Ref. Prou & Lieu d'émission & Monétaire & Ag \\
\hline 1431 & Marseille & - & $66,0 \%$ \\
\hline 1440 & Marseille & - & $61,6 \%$ \\
\hline 1444 & Marseille & "ISARNO" & $69,8 \%$ \\
\hline 1449 & Marseille & Antenor & $62,8 \%$ \\
\hline 1451 & Marseille & Ansedert & $58,5 \%$ \\
\hline 1473 & Marseille & Ansedert & $61,5 \%$ \\
\hline 1477 & Marseille & Ansedert & $74,9 \%$ \\
\hline 1482 & Marseille & Ansedert & $67,8 \%$ \\
\hline 1484 & Marseille & Ansedert & $65,1 \%$ \\
\hline 1518 & Marseille & Nemfidius & $63,4 \%$ \\
\hline 1529 & Marseille & Nemfidius & $68,0 \%$ \\
\hline 1546 & Marseille & Nemfidius & $58,0 \%$ \\
\hline 1569 & Marseille & Nemfidius & $43,4 \%$ \\
\hline 1586 & Marseille & Nemfidius & $57,1 \%$ \\
\hline 1615 & Marseille ? & - & $50,6 \%$ \\
\hline
\end{tabular}

Si l'on considère comme des corpus cohérents les lots de monnaies mérovingiennes analysés par D.M. Metcalf, J. Lafaurie, J. Poirier et J.-N. Barrandon, ainsi que celui constitué par le trésor de Rodez, il apparaît que les deniers d'argent frappés en Gaule entre 675 et 750 environ présentent des titres d'argent très

62. Les références et titres d'argent de ces deniers sont présentés en Annexe 1. Seule la pureté des pièces y apparaît : la méthode employée ne permettait pas le dosage des éléments mineurs et traces. Merci à J. Poirier et J.-N. Barrandon pour avoir réalisé ces analyses, et à M. Bompaire pour nous les avoir fait connaître. 
variables. La gamme de teneurs couverte par l'ensemble de ces pièces s'étend, à de rares exceptions près, de $40 \%$ à près de $100 \%$ d'argent (figure 20). La datation précise de la plupart des pièces du trésor de Rodez et de celles analysées par D.M. Metcalf et par J. Poirier et J.-N. Barrandon est malaisée, aussi nous nous limiterons à cette approche globale pour ces trois corpus. L'attribution à un évêque des deniers épiscopaux de Paris publiés par J. Lafaurie permet de proposer un suivi de l'évolution de la pureté des alliages d'argent de ce type au cours de l'intervalle durant lequel de telles pièces ont été frappées. La reprise de l'interprétation de J. Lafaurie sur les résultats obtenus, ainsi que la comparaison avec les valeurs qui caractérisent les deux deniers parisiens épiscopaux du trésor de Rodez, apparaît plus loin dans ce texte.

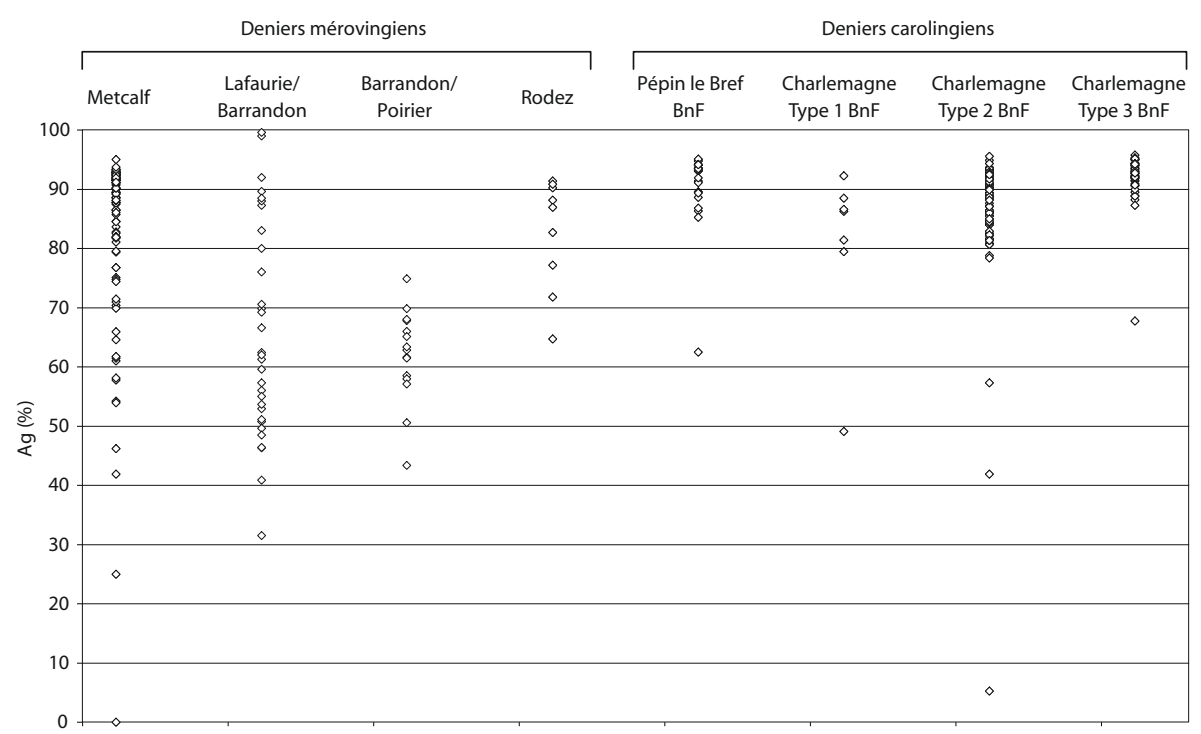

Figure 20 - Représentation graphique du titre d'argent des pièces du trésor de Rodez. Comparaison avec les résultats publiés par Metcalf et par Lafaurie, ceux non publiés de Poirier et Barrandon, et avec ceux concernant les pièces des premiers Carolingiens conservées à la $\mathrm{BnF}^{63}$.

La comparaison du titre d'argent des deniers mérovingiens avec celui des premières frappes carolingiennes permet d'approfondir l'interprétation de l'évolution de la pureté des alliages monétaires d'argent au VIII ${ }^{\mathrm{e}}$ siècle. Une première tendance peut être observée à l'examen de la figure 20, lors du passage du monnayage d'argent mérovingien au monnayage d'argent carolingien. Si, comme cela est probable, l'enfouissement du trésor de Rodez date de l'extrême fin de

63. Voir D.M. MetCALF, op. cit., n. 58, J. LAFAurie, op. cit., n. 38, p. 84, et G. SARAH, op. cit., n. 56. 
la période mérovingienne, voire des premières années de règne de Pépin le Bref, une comparaison peut être envisagée entre ces deux monnayages : les exemplaires du Cabinet des Médailles de la Bibliothèque nationale de France du premier souverain carolingien ont en effet été récemment analysés ${ }^{64}$.

La moyenne de titre d'argent pour les deniers mérovingiens supposés tardifs du trésor de Rodez est de $82,7 \%$, tandis que cette valeur est de $92,0 \%$ pour ceux de Pépin le Bref. Seule une des vingt-six monnaies analysées de ce souverain présente un titre inférieur à $85 \%$ d'argent - et l'écart important observé entre la pureté de cet exemplaire et celle de l'ensemble homogène formé par les autres incite à penser qu'il pourrait s'agir d'une imitation -, alors que c'est le cas pour quatre des neuf pièces exhumées à Rodez (figure 20). Les écarts-types relatifs calculés à partir des valeurs de titre confirment la variabilité plus grande de la composition des deniers du trésor de Rodez par rapport à ceux du premier souverain carolingien conservés au Cabinet des Médailles : ces valeurs, qui représentent la dispersion des titres d'argent des pièces considérées pour chacun de ces deux groupes, sont respectivement de $11,5 \%$ et de $3,1 \%$. Ces observations, si l'on se base sur l'hypothèse d'une continuité chronologique entre les deux monnayages considérés, et si les alliages constituant les neuf pièces du trésor de Rodez sont représentatifs de l'ensemble de ceux de la fin de la période mérovingienne, suggèrent que les premières frappes carolingiennes sont l'occasion d'un renforcement et d'une normalisation du titre d'argent des alliages monétaires. L'uniformisation des deniers du point de vue numismatique, avec la mention du nom de Pépin sur les pièces carolingiennes, ainsi que la réduction du nombre de lieux d'émission semblent confirmer cette tendance.

Les teneurs en argent déterminées pour les deniers du trésor de Rodez, comme pour ceux caractérisés antérieurement, couvrent de manière relativement homogène une gamme de teneurs très large, à l'exception d'un ou deux exemplaires étudiés par D.M. Metcalf qui ne contiennent pas ou très peu de métal précieux. Les pièces des premiers souverains carolingiens frappées à partir de 751 et jusqu'à la réforme de 793/794 ${ }^{65}$, présentent une variabilité de titre d'argent nettement moindre que celle observée pour les deniers mérovingiens : alors que l'écart-type relatif calculé pour les quatre premiers types monétaires carolingiens frappés entre 751 et 793/4 (Pépin le Bref, Charlemagne Type 1, Charlemagne Type 2, Charlemagne Type 3 ) est au maximum égal à $5 \%$, cette caractéristique

64. G. SARAH, op. cit., n. 56.

65. La chrono-typologie adoptée pour les premières monnaies carolingiennes est la suivante : Pépin le Bref (751-768); Charlemagne Type 1 (768-771); Charlemagne Type 2 (771-793/794); Charlemagne Type 3 (793/794-812). Sur le classement typologique des pièces de Pépin le Bref et de Charlemagne, ainsi que sur la datation de leur émission, voir Ph. Grierson, M. Blackburn, op. cit., n. 36, p. 190-217. Voir également Ph. Grierson, Money and coinage under Charlemagne, dans Karl der Grosse: Lebenswerk und Nachleben. Bd.1 : Persönlichkeit und Geschichte, 3. Auflage, Düsseldorf, 1967, p. 501-536 ; J. LAFAURIE, Numismatique : des mérovingiens aux carolingiens, Francia, 2, 1974, p. 26-48. 
est comprise entre $11 \%$ et $27 \%$ pour les quatre corpus de deniers mérovingiens considérés (19,1\% pour l'ensemble de ces pièces).

Une autre remarque peut être formulée à propos du titre d'argent des deniers mérovingiens et carolingiens d'après la figure 20 : elle concerne l'identification de monnaies fausses ou d'imitation. La continuité des valeurs de titre des deniers mérovingiens comprises entre les bornes minimale et maximale déterminées rend difficile l'identification de monnaies d'imitation. Seul un denier analysé par D.M. Metcalf, dont la composition est exempte d'argent ( $\left.\mathrm{n}^{\circ} 33\right)$, peut être exclu avec assurance du corpus des monnaies authentiques. La représentation graphique du titre d'argent pour les monnaies carolingiennes de Pépin le Bref et des trois premiers types monétaires de Charlemagne met en revanche en évidence la différenciation de quelques exemplaires de titre plus faible que la majorité des pièces de chacun de ces types. Il est alors possible d'envisager que ces pièces moins pures constituent des «faux» au sens large, imitations issues d'ateliers frauduleux ou des officines légales.

Les alliages monétaires à base d'argent contiennent différents constituants sous forme d'impuretés dénommés «éléments mineurs et traces» par l'analyste. Concernant les teneurs de ces constituants, plusieurs observations ressortent de l'étude de la composition des pièces d'argent du trésor de Rodez.

Les concentrations relativement élevées en étain, et dans une moindre mesure en zinc, suggèrent, selon les cas, l'utilisation de bronze ou de laiton pour affaiblir un alliage d'argent de pureté supérieure pour la plupart des neuf pièces étudiées. Cette tendance est confirmée par l'examen des rapports étain/ cuivre et zinc/cuivre (tableau 2). De telles valeurs ne sont pas observées pour les deniers de Pépin le Bref du Cabinet des Médailles. Il semble donc que l'augmentation de la pureté des pièces à la transition des monnayages mérovingiens et carolingiens se soit doublée d'une amélioration de la pureté des alliages cuivreux alliés à l'argent.

Parmi les éléments mineurs et traces présents en tant qu'impuretés dans les monnaies à base d'argent, certains peuvent apporter des informations sur l'origine du métal précieux utilisé. C'est le cas de l'or, dont les teneurs ont été déterminées lors de l'analyse des deniers du trésor de Rodez, ainsi que dans les recherches menées par D.M. Metcalf et par J. Lafaurie sur la composition des monnaies d'argent mérovingiennes.

L'ensemble des monnaies du trésor de Rodez présente des teneurs en or relativement élevées, de l'ordre de $1 \%$ le plus souvent et jusqu'à plus de $4 \%$, à l'exception de l'exemplaire 8 qui n'en contient que $0,54 \%$. Il est peu probable que de telles valeurs puissent résulter d'une chaîne opératoire minéralurgique et métallurgique d'extraction d'argent à partir de minerai. Plus vraisemblablement, de l'or aurait été mélangé à l'argent constituant les pièces mérovingiennes du trésor de Rodez. Des concentrations d'or aussi importantes peuvent être retrouvées dans le monnayage de Pépin le Bref et celui de Charlemagne antérieur à la réforme de 793/4 (Types 1 et 2). Elles sont en revanche inexistantes pour les frappes plus tardives (Type 3 ). Une hypothèse à considérer pourrait être 
la refonte de monnaies d'or mérovingiennes très altérées, et dont l'aspect aurait été proche de celui de l'argent, avec du métal blanc lors de l'introduction du denier mérovingien à la fin du vil ${ }^{\mathrm{e}}$ siècle. Les procédés d'affinage des métaux précieux au haut Moyen Âge permettant difficilement de séparer l'or de l'argent, on peut avancer l'hypothèse que le métal jaune contenu dans les deniers mérovingiens aurait subsisté dans la masse métallique monnayée durant le $\mathrm{VIII}^{\mathrm{e}}$ siècle jusqu'aux frappes carolingiennes de Pépin le Bref et aux premières de Charlemagne. C'est la répétition des refontes, conjuguée à une augmentation de la masse métallique en circulation que l'on peut situer autour du passage du Type 2 au Type 3 de Charlemagne durant l'hiver 793/794, qui pourrait avoir été à l'origine du phénomène observé. La décroissance des teneurs en or observée lors du passage du Type 2 au Type 3 de Charlemagne, correspondant à la réforme monétaire de 793/794, pourrait alors être due à une augmentation significative du numéraire en circulation. S'il ne s'agit que de conjectures, une continuité semble exister dans les teneurs en or entre les deniers mérovingiens tardifs du trésor de Rodez et certains des deniers carolingiens, légèrement postérieurs, de Pépin le Bref.

La représentation graphique des teneurs en or déterminées pour les trois corpus de deniers d'argent mérovingiens considérés dans cette partie (Metcalf, Lafaurie et trésor de Rodez) apparaît sur la figure 21, ainsi que la comparaison avec les premières pièces carolingiennes ultérieures. La première remarque qui peut être faite à l'examen de la figure 21 est la différenciation du point de vue des teneurs en or des monnaies du trésor de Rodez par rapport à celles dont les résultats ont été publiés par D.M. Metcalf et par J. Lafaurie. La représentation graphique des valeurs proposées par ces deux chercheurs met en évidence, dans les deux cas, un groupe homogène, caractérisé par des concentrations en or le plus souvent inférieures à $1 \%$. Les teneurs qui caractérisent les pièces du trésor de Rodez sont supérieures, voire très supérieures à celles de D.M. Metcalf et J. Lafaurie. L'interprétation de cette différence semble complexe. L'étude seule de l'évolution des teneurs en or déterminées par nos soins dans les monnaies mérovingiennes du trésor de Rodez, puis dans les carolingiennes de Pépin le Bref et des trois premiers types monétaires de Charlemagne, suggère une décroissance progressive des concentrations de cet élément, ou du moins la disparition des alliages les plus riches en métal jaune. Notre interprétation présentée dans le paragraphe précédent rejoindrait alors celle de J. Lafaurie - à la seule lumière d'un unique résultat - qui suggère la refonte de tremisses d'or mérovingiens fortement altérés pour la frappe de deniers ${ }^{66}$. Toutefois, si l'on

66. J. LAFAURIE, op. cit., n. 38, p. 83 : «Ce qui peut être retenu de ces constatations est que les ateliers monétaires devaient être équipés pour affiner les métaux mais que ces affinages, longs à effectuer, l'ont été plus ou moins sérieusement au moment où devait être fabriquée la masse de deniers remplaçant les monnaies d'or. Ces monnaies d'or, décriées, étant tombées à des titres fort bas, autour de 10\%, ont pu servir de matière première avec quelques ajouts d'argent pour ces fabrications ». 
considère l'ensemble des résultats analytiques obtenus par le passé pour des deniers mérovingiens, notre interprétation peut sembler infondée : qu'il s'agisse des données proposées par D.M. Metcalf ou par J. Lafaurie, aucune mise en perspective des concentrations en or ne peut être effectuée avec les pièces des premiers souverains carolingiens.

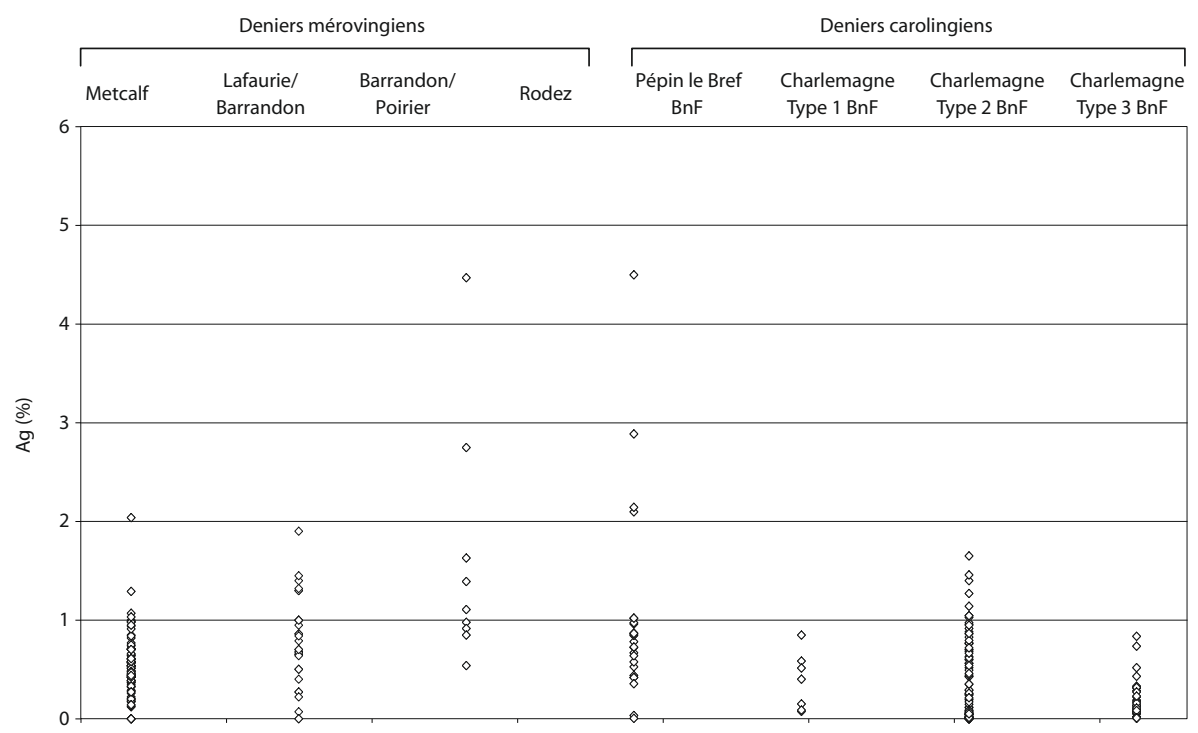

Figure 21 - Représentation graphique des teneurs en or des pièces du trésor de Rodez. Comparaison avec les résultats publiés par Metcalf et par Lafaurie, et avec ceux concernant les pièces des premiers Carolingiens conservées à la $\mathrm{BnF}^{67}$.

L'étude faite par J. Lafaurie des monnaies du trésor de Manre (Ardennes), à la fois du point de vue de la numismatique, de celui de leur aspect et de leur composition, doit également être considérée ici ${ }^{68}$ : quatre de ces pièces issues d'une sépulture, présentent, pour trois d'entre elles, un aspect d'argent pur, et correspondent à des types de tremissis; les analyses de composition effectuées par A.A. Gordus ont révélé des teneurs en or de $10 \%$ pour la monnaie jaunâtre, et de $0,9 \%$ à $6,5 \%$ pour les trois dont l'aspect est celui de l'argent. L'ensemble de ces données ne permet pas de trancher entre l'hypothèse de tremisses d'or très altérés, ou celle de deniers d'argent primitifs contenant beaucoup d'or résiduel.

67. Voir D.M. Metcalf, op. cit., n. 59, J. LAFAurie, op. cit., n. 38, p. 84, et G. SARAh, op. cit., n. 56. 68. J. LAFAURIE, Trouvailles de monnaies mérovingiennes à Manre (Ardennes), BSFN, 1 , 1972, p. 146-147. J. LAFAURIE, Les frappes monétaires de Metz et de sa région aux VI ${ }^{\mathrm{e}}-\mathrm{IX}^{\mathrm{e}}$ siècles, dans P. Riché, Actes du colloque Autour d'Hildegarde, Cahiers du Centre de recherches sur l'Antiquité tardive et le haut Moyen Âge, 1987, p. 89-103. 
Il semble toutefois que l'évolution typologique comme celle de la composition lors du passage d'un monnayage d'or à un nouveau d'argent autour de 675 ait été progressive. Ces observations concordent avec l'hypothèse d'une dilution progressive de l'or résiduel mérovingien dans la masse d'argent monnayée mérovingienne puis carolingienne.

Les corpus de deniers mérovingiens de D.M. Metcalf et de J. Lafaurie contiennent des exemplaires dont les types sont identiques ou similaires à certaines des pièces du trésor de Rodez. Trente-cinq des deniers mérovingiens analysés par D.M. Metcalf sont attribués à la civitas de Marseille. Parmi ces exemplaires, vingt peuvent être rapprochés du patrice de Provence Nemfidius, et auraient, selon cet auteur, été frappés au tournant du VII ${ }^{\mathrm{e}}$ au VIII ${ }^{\mathrm{e}}$ siècle ${ }^{69}$. Les deniers qui portent ce nom formeraient selon D.M. Metcalf un groupe distinct des autres exemplaires de Marseille d'une part, et des pièces d'autres cités d'autre part ${ }^{70}$. Trois pièces du corpus de D.M. Metcalf attribuables à Marseille $\left(\mathrm{n}^{\mathrm{os}} 44,45\right.$ et 46$)$ peuvent être rapprochées du denier 4 du trésor de Rodez issu de l'atelier de cette même cité ou plus sûrement de la région marseillaise - bien que l'orientation du visage au droit soit différente. Le denier du trésor étudié ici contient d'après notre analyse $91,4 \%$ d'argent. Cette valeur est très proche de celles déterminées pour les exemplaires référencés 44 et 45 par D.M. Metcalf (respectivement $92,42 \%$ et $91,94 \%$ ), mais assez éloignée du troisième du même type ( $\mathrm{n}^{\circ} 46,70,40 \%$ d'argent). Les teneurs en or sont en outre plus faibles que celle obtenue par nos soins pour le denier du trésor de Rodez de même type dont il est question dans ce paragraphe (de l'ordre de $0,5 \%$ contre $1,1 \%$ ). Le faible nombre de monnaies considérées, ainsi que les éventuelles incertitudes liées à l'utilisation d'une méthode d'analyse superficielle par D.M. Metcalf, nous incitent à la prudence ; aussi aucune interprétation ne sera risquée à partir de ces quelques résultats.

Les attributions d'une part importante des deniers mérovingiens épiscopaux de Paris à des évêques précis permettent de proposer, le plus souvent, un intervalle pour leur date d'émission. J. Lafaurie a ainsi pu étudier l'évolution de la pureté des pièces d'argent mérovingiennes frappées au nom d'évêques de Paris analysées par J.-N. Barrandon dans le cadre de leur collaboration, et en proposer une interprétation. Ce travail a permis de mettre en évidence deux phases distinctes au cours de la période d'émission des pièces d'argent mérovingiennes ${ }^{71}$ - avec les limites liées au faible nombre d'exemplaires étudiés pour certains évêques, et les incertitudes quant à l'attribution d'une partie des deniers à certains de ces prélats. J. Lafaurie relève en premier lieu une période comprise entre l'introduction du denier d'argent mérovingien vers 675 et la fin de l'épiscopat de Turnoaldus en 717 , durant laquelle la pureté des alliages constituant les

69. D.M. MetCalF, op. cit., n. 58, p. 114.

70. Ibid.

71. J. Lafaurie, op. cit., n. 38, p. 83. 
deniers aurait été élevée ou très élevée : les teneurs en argent déterminées pour les pièces attribuées aux évêques dont les épiscopats appartiennent à cet intervalle sont comprises entre $83 \%$ et $99 \%$. Le titre des monnaies d'argent aurait chuté durant les décennies suivantes : les valeurs obtenues par J.-N. Barrandon pour J. Lafaurie sont comprises entre $40 \%$ et $70 \%$ pour ceux attribués aux évêques Adulfus, Berneharius, Hugo, Merfridus et Ragnecapdus, dont les frappes peuvent être datées des années 720 à 750 environ. Cette seconde période aurait été marquée par une hausse ponctuelle du titre des deniers durant l'épiscopat de Ratbertus selon J. Lafaurie. La représentation graphique de ces résultats sur la figure 22 suggère plutôt selon nous le passage d'une première période durant laquelle le titre des deniers est élevé ou très élevé, à une seconde marquée par une chute de la teneur moyenne en argent et une augmentation significative de la variabilité des valeurs. La hausse ponctuelle durant l'épiscopat de Ratbertus observée par J. Lafaurie doit être relativisée vu le faible nombre d'analyses de deniers de chaque évêque présentées, et ne peut être considérée avec assurance comme une tendance qui reflète la réalité de l'évolution de la composition des deniers épiscopaux de Paris. J. Lafaurie mentionne à juste titre la nécessité de poursuivre les analyses de deniers épiscopaux de Paris pour confirmer les tendances observées. Seule une campagne d'analyse de deniers mérovingiens de grande ampleur, incluant un nombre conséquent de deniers épiscopaux parisiens, pourrait en effet éclaircir cette problématique.

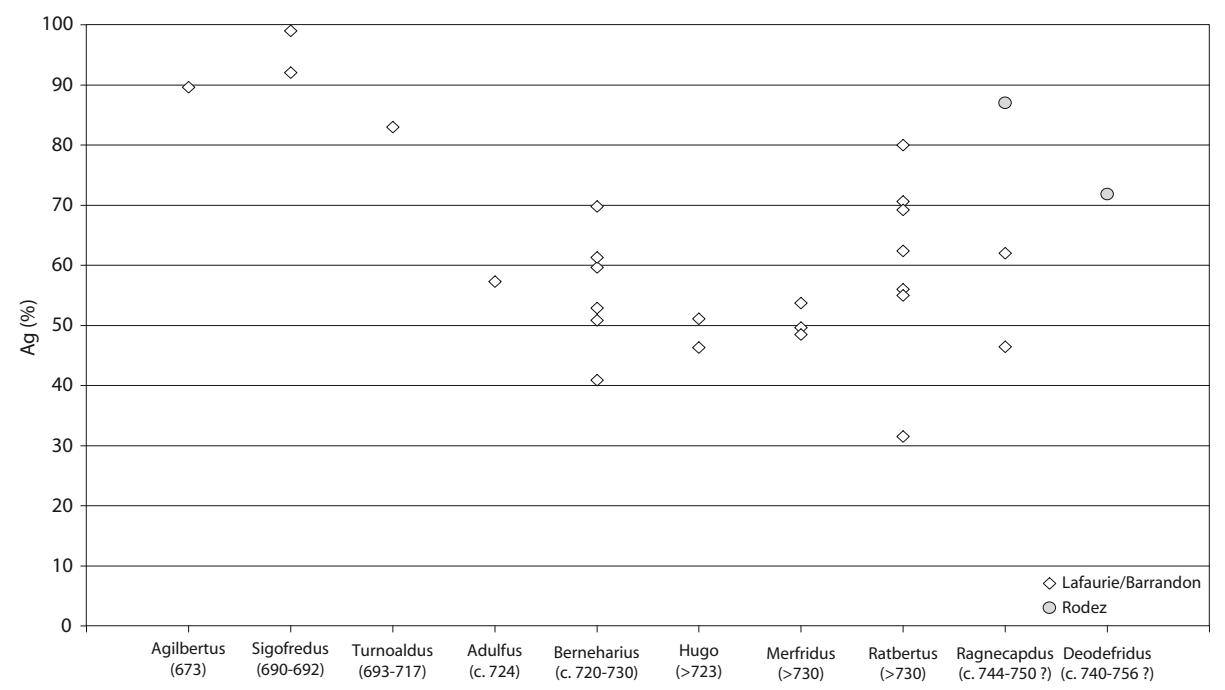

Figure 22 - Représentation graphique de l'évolution du titre d'argent des deniers mérovingiens frappés par les évêques de Paris. Résultats d'après nos analyses et celles de Lafaurie ${ }^{72}$.

72. J. LAFAURIE, op. cit., n. 38, p. 84. 
Deux deniers du trésor de Rodez ont été attribués à des évêques de Paris d'après leur étude numismatique : celui référencé 5 serait de Ragnecapdus, tandis que le $\mathrm{n}^{\circ} 6$ pourrait avoir été frappé durant l'épiscopat de Deodefridus. La représentation graphique du titre d'argent de ces deux deniers apparaît sur la figure 22, sur laquelle sont également reportées les valeurs déterminées par ANRC (Activation aux Neutrons Rapides de Cyclotron) par J.-N. Barrandon et qui apparaissent dans la publication de J. Lafaurie. L'apport de ces deux nouveaux résultats à la compréhension globale de la frappe des deniers épiscopaux à Paris durant la période mérovingienne est bien entendu minime. Aucune conclusion ne peut être tirée de ces deux seuls résultats quant à la pureté des deniers frappés aux noms de Ragnecapdus et Deodefridus. On peut néanmoins relever que la pièce $\mathrm{n}^{\circ} 5$ du trésor de Rodez présente un titre nettement plus élevé que les deux exemplaires de la $\mathrm{BnF}$ dont les résultats sont publiés par J. Lafaurie, Prou 757 et Prou 758 (87,0\% contre respectivement $62,0 \%$ et $46,4 \%)$. Cet écart est d'autant plus remarquable que la liaison de coin qui semble exister entre le denier récemment mis au jour à Rodez et Prou 757 tend immanquablement à les rapprocher sur le plan chronologique.

Les deux points correspondant à nos analyses (figure 22) tendent à modifier l'allure générale de la courbe d'évolution qui était dessinée par les seuls résultats de J. Lafaurie : il semble d'après la combinaison de toutes les valeurs prises en compte qu'une hausse du titre d'argent des deniers ait suivi la chute relevée par J. Lafaurie. Plus qu'une réelle tendance, cette modification de l'interprétation de la représentation graphique de la pureté des deniers épiscopaux de Paris sur la seule base de deux résultats supplémentaires nous incite à penser que le nombre d'analyses prises en compte demeure insuffisant. Si l'on considère de surcroît les incertitudes relatives aux difficultés d'attribution de ces pièces, une interprétation approfondie de ces résultats semble risquée et prématurée en l'état.

L'étude conjointe des types numismatiques et de la composition des alliages monétaires apporte des éléments de réflexion nouveaux pour la compréhension et la datation du trésor de Rodez, et plus largement pour les émissions d'argent mérovingiennes. Malgré leur faible représentativité, l'ensemble de ces données encourage donc à systématiser les analyses de deniers mérovingiens, en particulier pour les exemplaires aux noms des évêques de Paris. 